\title{
L'habitat moustérien de « La Folie » (Poitiers, Vienne) : synthèse des premiers résultats
}

Mousterian spatial organisation in the site of La Folie (Poitiers, Vienne):

Synthesis of the first results

L. Bourguignon, F. Sellami, V. Deloze, N. Sellier-segard, S. Beyries et A. Emery-barbier

\section{OpenEdition}

\section{Journals}

Édition électronique

URL : http://journals.openedition.org/paleo/1389

DOI : $10.4000 /$ paleo.1389

ISSN : 2101-0420

\section{Éditeur}

SAMRA

\section{Édition imprimée}

Date de publication : 1 décembre 2002

Pagination : $29-48$

ISSN : $1145-3370$

\section{Référence électronique}

L. Bourguignon, F. Sellami, V. Deloze, N. Sellier-segard, S. Beyries et A. Emery-barbier, « L'habitat moustérien de «La Folie » (Poitiers, Vienne) : synthèse des premiers résultats », PALEO [En ligne], 14 2002, mis en ligne le 10 août 2010, consulté le 22 juillet 2020. URL : http://journals.openedition.org/ paleo/1389; DOI : https://doi.org/10.4000/paleo.1389

Ce document a été généré automatiquement le 22 juillet 2020.

\section{cc) (†)}

PALEO est mis à disposition selon les termes de la licence Creative Commons Attribution - Pas d'Utilisation Commerciale - Pas de Modification 4.0 International. 


\section{L'habitat moustérien de « La Folie » (Poitiers, Vienne) : synthèse des premiers résultats}

Mousterian spatial organisation in the site of La Folie (Poitiers, Vienne):

Synthesis of the first results

L. Bourguignon, F. Sellami, V. Deloze, N. Sellier-segard, S. Beyries et A. Emery-barbier

\section{I- Introduction}

1 La fouille de sauvetage urgent du site Paléolithique moyen de "La Folie" a été réalisée dans le cadre d'un projet de construction d'une station d'épuration par la communauté urbaine de la ville de Poitiers. Le gisement est localisé au nord de la commune de Poitiers, au lieu-dit "La Folie”, non loin de la zone industrielle République (Fig. 1a). 
Fig. 1a - Carte de localisation du site Fig. 1 a - Location of the site

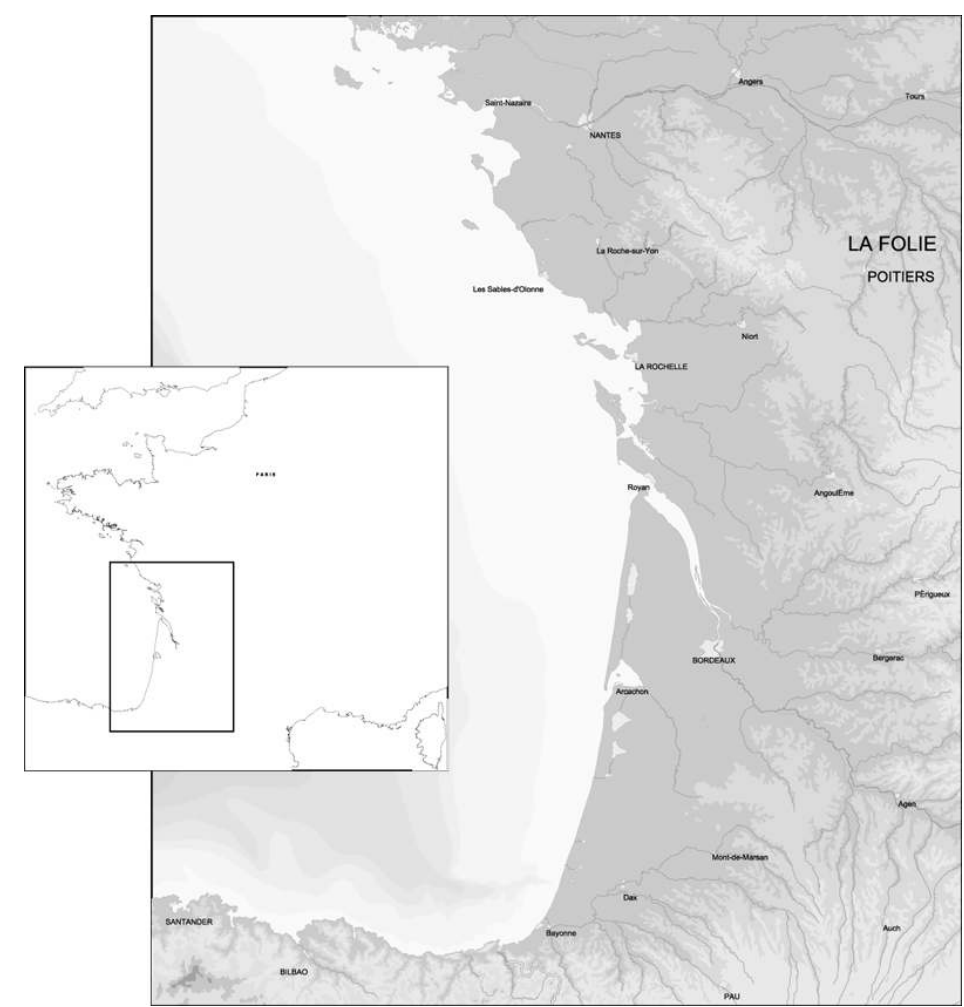

2 L'étendue du site paléolithique de "La Folie" à Poitiers se limite à la zone de décapage qui révèle la présence d'une surface d'occupation fouillée dans son intégralité sur $580 \mathrm{~m}^{2}$ (Fig. 1b). Cette zone est structurée par deux grands amas de débitage à l'est et à l'ouest et une organisation concentrique de blocs calcaires d'origine anthropique marquant l'emplacement probable d'une surface d'habitation (cf. infra). 
Fig. 1b - Surface de fouille du gisement moustérien de La Folie Fig. $1 b$ - Excaved area of the mousterian site of La Folie

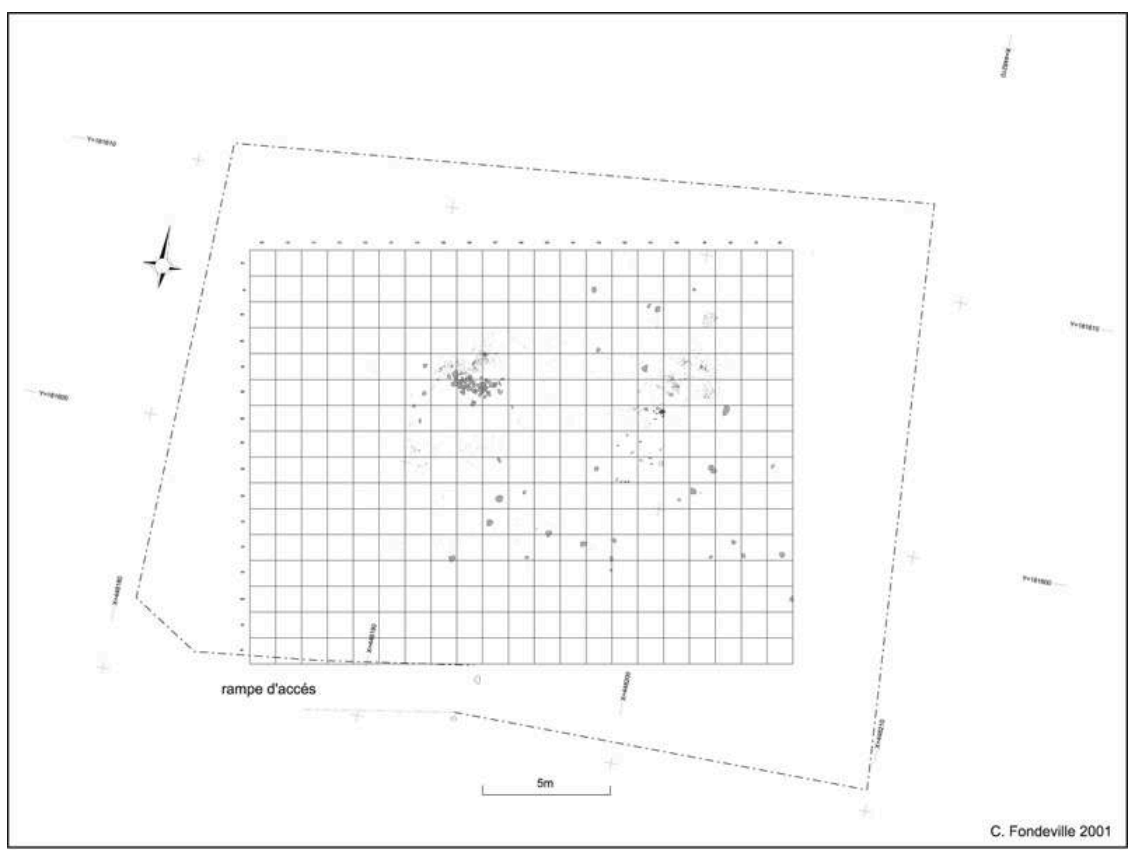

3 La particularité du site réside dans la préservation exceptionnelle du matériel archéologique qui a peu souffert des phénomènes d'altération post-dépositionnels. Le seul niveau archéologique de faible épaisseur $(10 \mathrm{~cm})$ a pu être traité dans sa totalité comme une unité (Fig. 2). Les remontages entre les secteurs est et ouest confortent cette interprétation ( $c f$. infra).

Fig. 2 - Coupe du niveau archéologique sur une bande d'un mètre sur l'axe transversal de l'occupation

Fig. 2 - Vertical distribution of all flint artefacts recoarded in a transversal axes by un $m$ trench

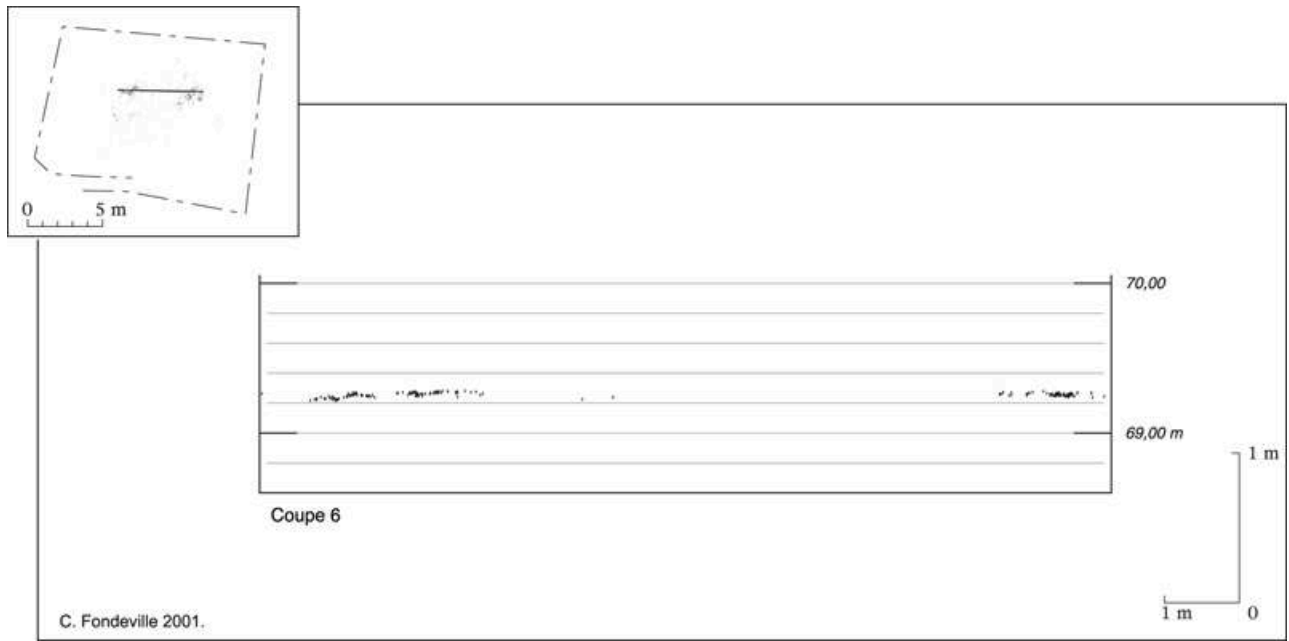




\section{II- Cadre géologique et géomorphologique}

4 Le site de «La Folie » est localisé sur la rive gauche du Clain à $3 \mathrm{~km}$ en aval de Poitiers. A cet endroit, les plateaux, dont les assises calcaires sont entaillées par quelques vallées sèches, culminent vers $125 \mathrm{~m}$ NGF et dominent la rivière de $60 \mathrm{~m}$. La vallée présente un profil contrasté avec, en rive droite, un coteau vertical contre lequel bute le cours actuel du Clain et en rive gauche, un versant à pente plus douce où subsiste un lambeau de nappe alluviale (Fig. 3). Ce versant est incisé par deux petits vallons secs rectilignes, très courts et d'axe ENE/WSW.

\section{1- Méthodologie}

Cette étude a été effectuée en trois étapes, lors des phases de prospection, évaluation et fouille (d'avril à novembre 2000), avec relevés de sondages géologiques complémentaires au fur et à mesure et réalisation d'une campagne de prélèvements malacologiques, palynologiques, micromorphologiques et sédimentologiques.

6 Le sondage 1 qui a conduit à la découverte du site lors de la prospection, a permis de relever en détail la coupe 1 de référence (Fig. $4 \mathrm{~b}$ ). Etendu progressivement lors des phases suivantes, il a abouti à un décapage de forme rectangulaire, dont l'axe le plus long est quasi est-ouest. Les trois autres côtés de ce décapage profond ont aussi été relevés en détail (Fig. 3, 4a et $4 \mathrm{~b}$ ), excepté dans les parties éboulées ou talutées. Les autres données stratigraphiques ont été fournies par les sondages géotechniques et par les coupes relevées dans les sondages 2, 3, 6, 30 et 31 .

Les premiers résultats, très résumés ici, correspondent à la description synthétique des treize niveaux stratigraphiques distingués sur les neuf coupes, en intégrant les résultats des analyses granulométriques et calcimétriques, et aux premières hypothèses d'attribution chronostratigraphique qui ont été émises (Deloze 2001). 
Fig. 3 - Coupe synthétique du Clain d'après les données géotechniques de la rocade Fig. 3 - Synthetic profil of the Clain valley

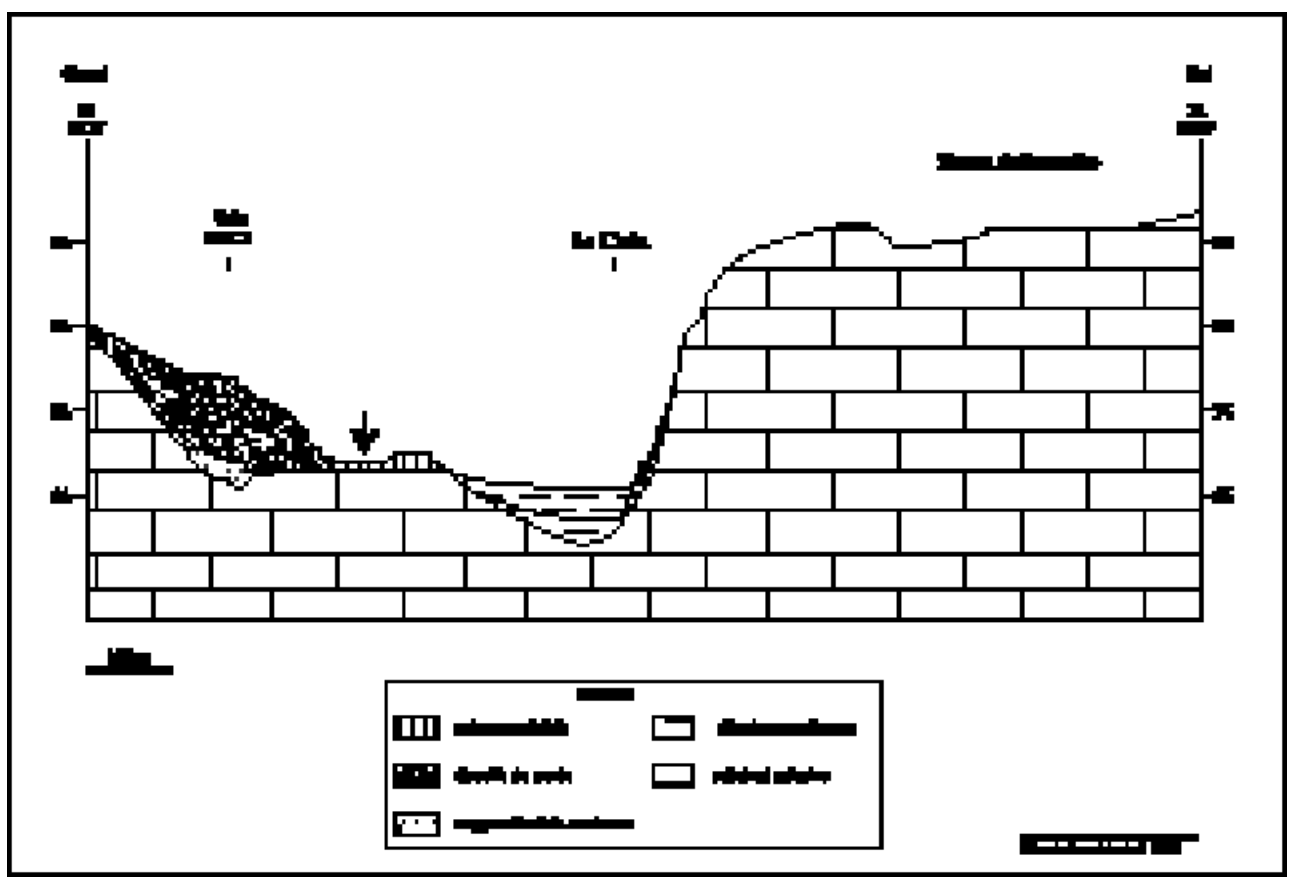

Fig. 4 - Coupes stratigraphiques du gisement ( $a$ et b) et représentation schématique de la coupe et des ensembles sédimentaires avec positionnement des échantillons étudiés (c)

Fig. 4 - Stratigraphic profil of the prehistoric site of La Folie ( $a$ and $b$ ) ans schematic representation of sedimentary horizon with samples position (c)
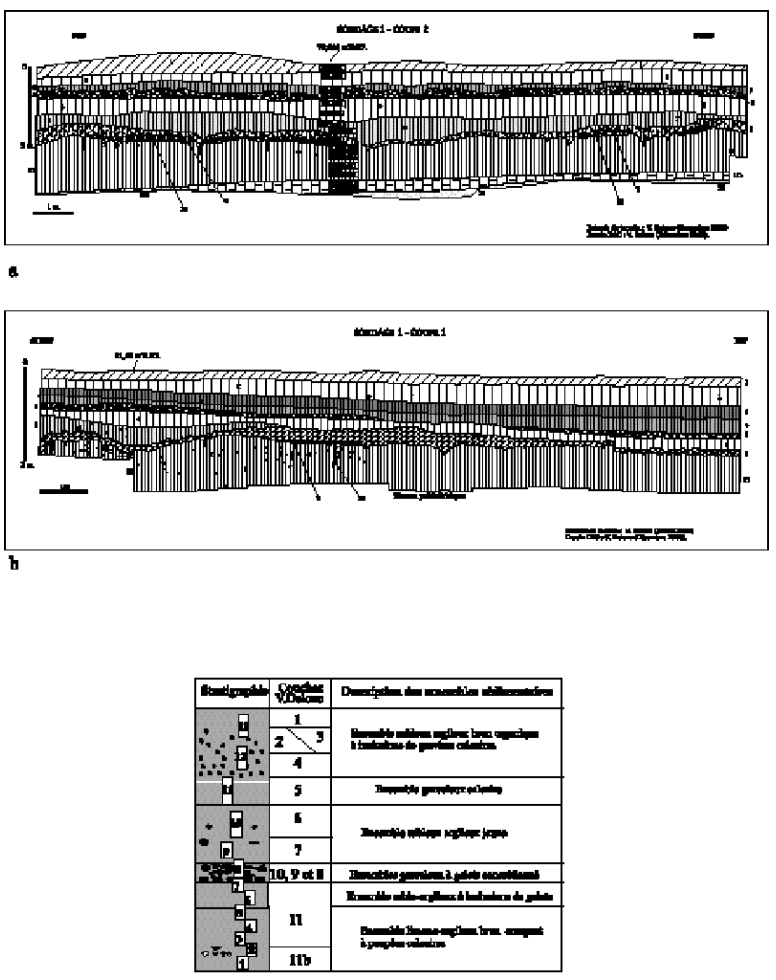


\section{II.2- Description de la séquence stratigraphique}

De haut en bas, les neuf relevés géologiques effectués sur le site de "La Folie" ont permis de distinguer les couches suivantes, numérotées de 1 à 13 (Fig. $4 \mathrm{a}$ et 4b) :

- couche 1 : terre végétale limoneuse brun foncé décarbonatée, contenant $20 \%$ de fragments de silex et granules calcaires et $20 \%$ de sables. Teinte 10 YR 4/3 (1);

- couche $2:$ limon décarbonaté à $4 \%$ de silex et de granules calcaires. Teinte 5 YR 4/4;

- couche 3 : limon décarbonaté contenant de rares éléments grossiers. Teinte 5 YR _;

- couche 4 : limon sableux décarbonaté à $40 \%$ de granules calcaires. Teinte 5 YR 4/4;

- couche $5: 17,4 \%$ de graviers et $17,4 \%$ de sables grossiers dans une matrice limoneuse carbonatée. Teinte 7,5 YR 6/6;

- couche 6 : limon sableux carbonaté à nombreux filaments calcaires et bioturbations, et contenant $10 \%$ d'éléments grossiers. Teinte 10 YR 5/8;

- couche 7 : limon assez fin carbonaté à nombreux filaments calcaires. Niveau parfois compact et induré au contact de la couche 8. Poupées calcaires et graviers épars. Teinte 10 YR 6/6;

- couche $8: 80 \%$ de cailloutis moyen (module $<7 \mathrm{~cm}$ ) composé de silex gris-bleuté et de calcaire, dans un ciment carbonaté parfois induré. Teinte hétérogène blanc-gris. Lit très irrégulier et géliturbé ;

- couche 9 : limon sableux carbonaté. Teinte 5 YR 6/6 ;

- couche 10 : cailloutis moyen (module $<7 \mathrm{~cm}$ ) de silex gris-bleuté et de calcaire, à ciment carbonaté et parfois induré. Teinte hétérogène blanc-gris. Lit très irrégulier et géliturbé ;

- couche 11 : limon fin carbonaté à nombreux filaments et poupées calcaires (2 à $4 \%$ ). Teinte 5 YR 6/6. Quelques passées carbonatées de teinte 10 YR 6/6 ;

- couche $11 \mathrm{~b}$ : limon argileux carbonaté à rares filaments et poupées calcaires. Teinte 5 YR 6/6;

- couche 12 : nappe alluviale sableuse brun-rouge à petits graviers alluviaux et nombreuses coquilles de gastéropodes ;

- couche $12 \mathrm{~b}$ : nappe alluviale gravelo-sableuse plus grossière et très meuble ;

- couche 13 : substrat calcaire du Callovien.

\section{II.3- Premières hypothèses d'interprétation et d'attribution chronostratigraphique}

9 A la base de la séquence, le substrat calcaire (13) apparaît d'aspect fracturé et altéré au contact des formations quaternaires (couches 1 à 12); il présente un toit à profil irrégulier, à chenaux étroits et encaissés.

Les couches 12 (Fig. $4 \mathrm{a}$ ) et $12 \mathrm{~b}$ correspondent à la partie basale grossière de la nappe alluviale Fy du Clain, épaisse selon les endroits de 0,4 à 3,5 m. Elle comprend des dépôts gravelo-sableux à la base (12b), à rares stratifications obliques incurvées et à précipitations ferro-manganiques ainsi que des dépôts sableux massifs au sommet (12), déposés par un cours d'eau à chenaux anastomosés (Campy et Macaire 1989). D'après les données cartographiques (Bourgeuil et al. 1976; Mourier et al. 1986), cette nappe serait datée du Weichsélien ancien (Pléistocène supérieur) et si on se réfère au tableau chronologique établi par Lebret et al (1993), elle pourrait se placer aux stades isotopiques $5 \mathrm{~d}$ à $5 \mathrm{~b}($ ?). 
Fig. 5 - Explication schématique de la formation des assemblages pédosédimentaires caractérisant le support du bloc de pierre dans le carré BB99

Fig. 5 - Schematic explication of pedosementar horizon formation caracterizing the stone support in BB99

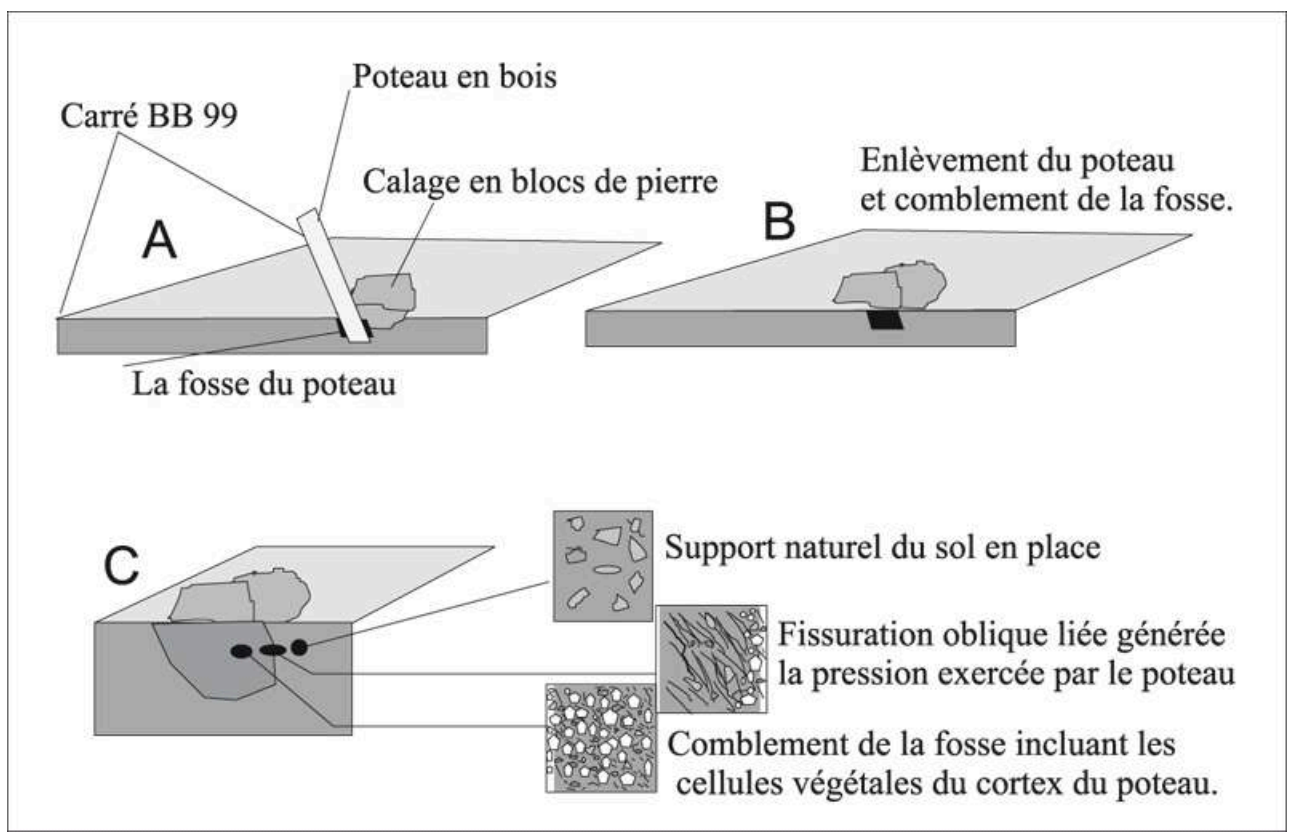

11 Les limons carbonatés jaune-rouge des niveaux 11 et $11 \mathrm{~b}$ (Fig. $4 \mathrm{a}$ et $4 \mathrm{~b}$ ), épais de 0,6 à $2,2 \mathrm{~m}$, se caractérisent par la présence de $25 \%$ de sables, d'accumulations carbonatées isolées (dues à la pédogenèse et à la dessiccation) et localement de lentilles plus grossières. Ils correspondent à des limons fins de débordement, mis en place dans sa plaine d'inondation par un cours d'eau à chenal unique et à méandres, dont les eaux ont une vitesse réduite et une importante charge sédimentaire fine (Campy et Macaire 1989). Cette séquence alluviale pourrait s'être mise en place sous conditions tempérées (peut-être interglaciaires?), peut-être à la fin du Weichsélien ancien (stade 5a?). L'unité domestique de "La Folie", datée du Paléolithique moyen, est localisée à la base de ces limons de débordement, témoignant d'une occupation suffisamment prolongée et temporairement à l'abri des crues (Deloze 2001).

12 Après une phase d'érosion, ces alluvions fines ont été recouvertes par un ou deux lits discontinus de cailloutis moyen de silex gris-bleu et de calcaire (8 et 10), entre lesquels s'intercalent parfois un limon sableux rouge-jaune à ciment carbonaté (9). Leurs matériaux provenant des affleurements de nappe ancienne et de colluvions situés en amont, ont transité sur le versant à la faveur du vallon sec repéré au sud-ouest du site. Il s'agit vraisemblablement de colluvions grossières, mises en place avec le concours des eaux de ruissellement et peut-être aussi des phénomènes de solifluxion, sous climat périglaciaire. Ces dépôts sont postérieurement marqués par des déformations dues au gel (présences de fentes de gel), des perturbations racinaires et une phase d'érosion.

La stratigraphie s'achève par une épaisse séquence de colluvions fines, parfois fortement érodées à leur sommet et souvent affectées par le gel et les phénomènes de 
décarbonatation ou recarbonatation. Difficilement datable, cette couverture est composée de :

- deux phases colluviales (6 et 7), à granulométrie nettement plus fine que les colluvions précédentes et représentées par des limons sableux brun-jaune dont l'épaisseur décroît du haut vers le bas du versant ;

- une phase de dépôts à petits granules calcaires anguleux emballés dans une matrice sablolimoneuse jaunâtre carbonatée (5), résultant de phénomènes cryoclastiques qui ont affecté le substrat calcaire gélif sur le versant, mis en place probablement sous climat périglaciaire ; - enfin, trois phases de colluvions, limoneuses à limono-sableuses, renfermant des granules calcaires cryoclastiques (couches 2, 3 et 4). Elles ont été piégées dans un paléorelief en creux aujourd'hui comblé et quasi invisible dans la topographie, situé presque dans le prolongement d'une vallée sèche.

14 A côté du site paléolithique, des structures datées du Néolithique (Tcheremissinoffet al. 2000 ; Tcheremissinoff et al. 2001) ont été mises au jour pour l'essentiel au sommet de la couche 5 , ce qui permet de rattacher cette phase de dépôts à une période antérieure (début de l'Holocène ?) et les colluvions sus-jacentes à l'Holocène sensu stricto.

\section{4- Conclusions}

Dans l'hypothèse où les corrélations et attributions chronostratigraphiques seraient pertinentes, l'unité domestique de "La Folie " datée du Paléolithique moyen pourrait se situer dans le stade 5a, soit entre 84000 et 72000 BP.

L'excellente conservation des artefacts paléolithiques serait la conséquence d'un emplacement particulièrement favorable dans la vallée du Clain sur le bord externe de la nappe alluviale $\mathrm{Fy}$, au sein de limons fluviatiles sus-jacents à une nappe alluviale non altérée, auxquels se superpose une couverture conséquente de dépôts où prédomine la dynamique de versant.

Malgré les nombreuses phases d'érosion, les témoins de l'occupation ont été conservés en place, même si, à cet endroit, on peut noter une légère dépression chenalisée correspondant en amont sur le versant, au prolongement d'une vallée sèche. Celle-ci a d'ailleurs pu servir de «couloir » d'évacuation des colluvions, des dépôts de versant et autres ruissellements, vers la vallée du Clain. 
Fig 6 - Intégration de matière argileuse au sein de la masse de cellules calcifiées Fig. 6 - Clayey material integration in the calcificaded cell
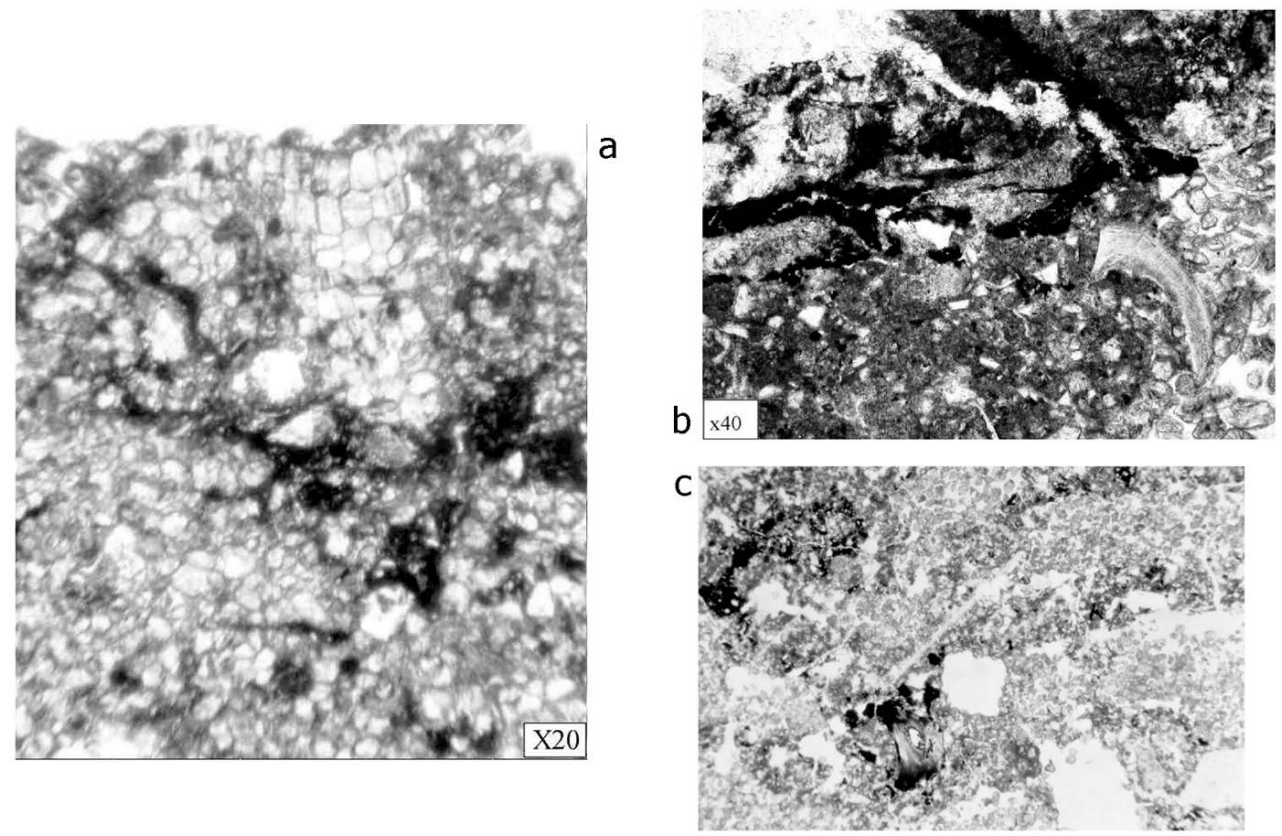

(a), plages charbonneuses résiduelles suite au lessivage du foyer (b) et fragments d'os brûlé intégré dans les cellules calcitisées au sein des taches noires (c)

(a), residual charcoal areas and fragments of burned bones in calcitised cell 
Fig. 7 - Dégradation en place de fragment végétal et conservation du réseau cellulaire Fig. 7 - Vegetal fragment degraded in situ and cell conserved
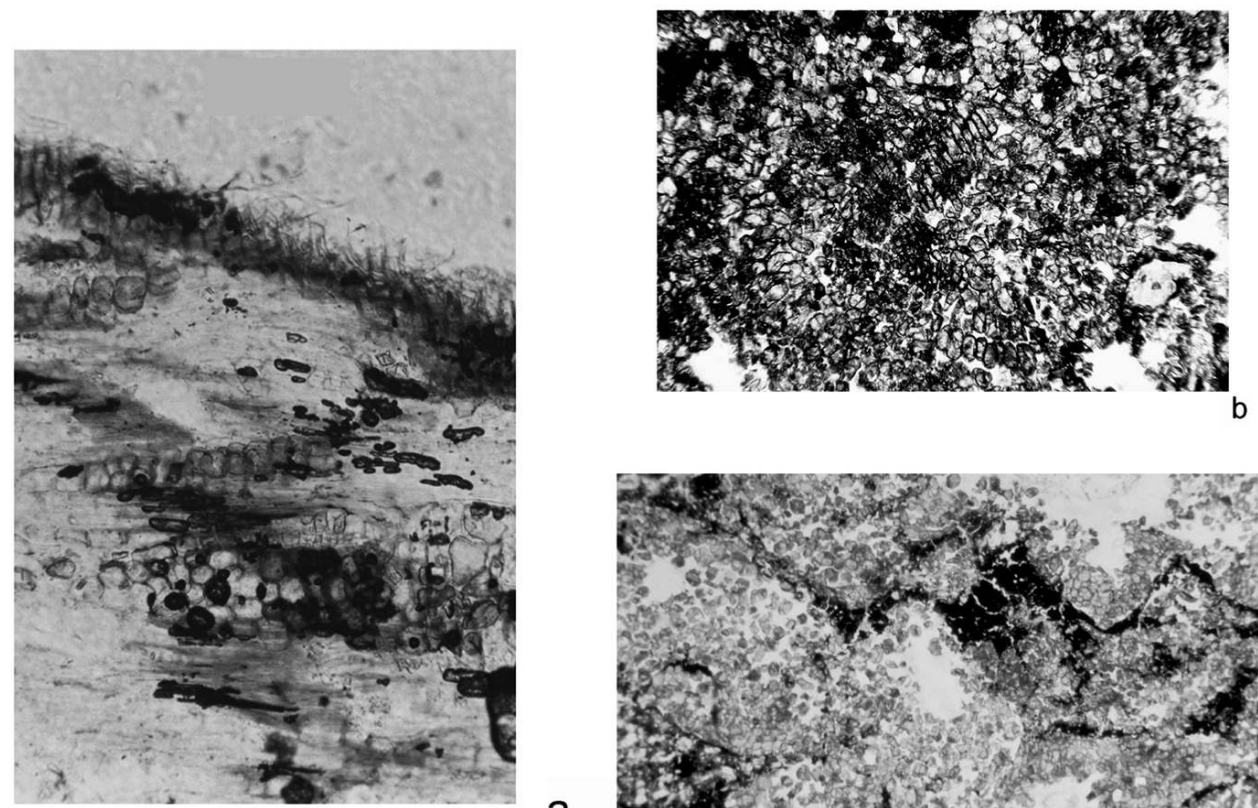

a

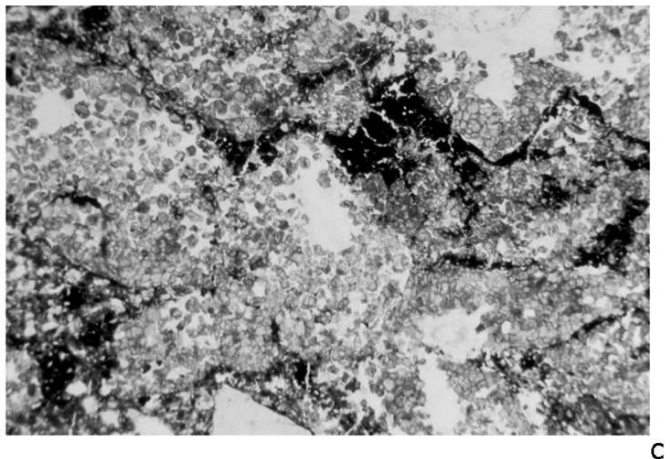

(a) réseau cellulaire organisé, calcifié et relativement bien conservé (b) et effondrement local du réseau cellulaire calcifié suite à la percolation d'eau (c)

(a), cells organised calcificaded and relatively well conserved (b) and local effondrement of calcificaded cell according to watter percolation

\section{III- Analyse micro-morphologique du niveau d'occupation}

Les limons d'inondation de la vallée du Clain ont permis le développement de sol brun calcaire marqué par une abondance de poupées calcaires en place. Enfoui à plus de trois mètres de profondeur, ce sol a révélé un degré d'enregistrement pédo-sédimentaire (Fig. 4c) exceptionnel qui s'est traduit par une bonne conservation des artefacts et de leur organisation. La préservation d'un niveau moustérien au sein de l'horizon profond a montré une occupation en place matérialisée par des assemblages de silex et par une organisation circulaire délimitée par des blocs de pierres en majorité calcaires. La surface du sol d'occupation se caractérise par des assemblages pédologiques marqués par des taches noires et diffuses pouvant être confondues avec des imprégnations de fer et par une plage blanchâtre rappelant les traces d'altération et de désagrégation en place d'ossements. Les investigations micro-morphologiques ont porté essentiellement sur l'analyse de sédiments provenant de la zone placée sous les blocs de pierre et de matériaux des assemblages pédologiques (taches noires ou blanchâtres) visibles sur le terrain.

Les résultats de l'étude sont de deux types, déterminant l'origine de l'organisation des blocs de pierre et définissant l'anthropisation de la surface du sol à laquelle s'ajoute la présence des amas de silex. 
20 L'analyse pédologique du sol supportant les blocs de pierre a montré un creusement bien délimité, comblé ultérieurement par l'effondrement partiel de ses parois sous l'effet de la saturation du sol en eau. La matrice sédimentaire de ce comblement est de ce fait partiellement lavée et la masse du sol est orientée, ce qui la différencie nettement de sol encaissant naturel adjacent. Ce dernier présente une structure légèrement compacte limitée aux abords de ce creusement. Son remplissage se caractérise par la présence de réseaux de cellules végétales en connexion conservées par calcitisation. Strictement limités au comblement du creusement, ces végétaux ne correspondent aucunement aux débris de décomposition de racines. La présence d'un creusement est confirmé par la combinaison de critères : la compacité de la paroi, la dégradation de restes végétaux notamment de l'épiderme (Freytet 1992 et com. orale) et l'apport des blocs de pierres. Tout ceci, nous laisse fortement à penser que le fonctionnement de cette structure correspond à celui des trous de poteau (Fig. 5).

21 L'étude des matériaux de certains assemblages pédologiques visibles à l'œil nu (tâches noires ou blanchâtres) et localisés sur la surface d'occupation a permis d'une part leur identification, d'autre part la reconstitution des processus de leur formation.

22 Ainsi, les assemblages blanchâtres en bordure nord de la concentration I a et b (Fig. 16) se sont révélés être des accumulations calcitiques déposées dans des cellules d'un fragment végétal dégradé en place (bois). Le réseau cellulaire est préservé grâce à une consolidation liée à la calcitisation. Son emballage sédimentaire est caractérisé par la présence d'abondants charbons associés à des fragments brûlés de la matrice du sol reflétant une zone de combustion. Sa partie superficielle, lessivée, et l'organisation de la sole ont été partiellement réorganisées par les processus biologiques. Tout ceci suggère l'existence d'un foyer altéré avec des restes de bois (fig.6).

Les taches noires, quant à elles, sont finalement limitées non seulement sur un plan horizontal, mais aussi et surtout sur un plan vertical. Leur formation n'est pas liée à la ferruginisation des sédiments. Elles sont issues exclusivement de la décomposition de matière organique végétale formant un lit relativement continu. Ce lit est emballé dans une matrice carbonatée où plusieurs réseaux de cellules végétales sont conservés par calcitisation. La limite de cet assemblage à environ cinq $\mathrm{cm}$ d'épaisseur et l'absence de réseau racinaire ont permis d'écarter l'hypothèse d'une dégradation d'un végétal développé naturellement. Bien au contraire, l'ensemble des critères pédologiques précédemment évoqués font penser à une accumulation de végétaux décomposés sur place. Bien qu'il soit difficile de préciser l'origine anthropique directe de cette accumulation, l'épaisseur homogène des réseaux cellulaires (environ cinq $\mathrm{mm}$ ), permet d'évoquer la présence porbable d'un seul et même végétal. Dans ce contexte, l'hypothèse d'une sélection anthropique de ces végétaux est possible. Nous envisageons la réalisation d' analyses complémentaires afin de déterminer la nature de la matière organique et surtout de rechercher d'éventuelles traces d'apports anthropiques expliquant ces accumulations végétales (Fig. 7) où la présence de restes osseux brûlés a été déjà attestée.

\section{IV- Industrie lithique}

L'industrie lithique moustérienne de "La Folie", bien que d'effectif modeste (1304 pièces en silex, coordonnées, dont 399 inférieures à $3 \mathrm{~cm}$ ), exprime clairement les 
comportements techniques et économiques des hommes implantés sur cette rive gauche du Clain.

Tout d'abord, en ce qui concerne l'acquisition des matières premières lithiques, ces comportements se caractérisent par une sélection de blocs bathoniens dans un territoire local, voire de proximité immédiate dans les dépôts de versants. Malgré une implantation sur les berges du Clain, les moustériens n'ont pas exploité les potentialités naturelles qu'offre ce milieu (galets alluviaux quel que soit le type de roche).

Seuls treize blocs de silex ont, semble-t-il, été nécessaires aux activités menées durant l'occupation. Leurs morphologies, variables (aplaties, plus ou moins circulaires ou sous forme de grosses plaques), ont généré des comportements techniques différents bien que tous orientés vers l'obtention de produits Levallois d'assez grandes dimensions. Ces adaptations se traduisent par la présence de plusieurs chaînes opératoires Levallois de modalités récurrentes. De même, occasionnellement, lorsque les conditions étaient réunies, des supports préférentiels ont été produits en début ou en fin de chaîne opératoire (Fig. 8). Les modalités d'aménagement et d'exploitation des blocs sont conjoncturelles. À une morphologie allongée du bloc correspondent des modalités récurrentes unipolaires et bipolaires (Fig. 9), à une morphologie sub-circulaire, une modalité centripète. Au sein d'un même bloc, les modalités peuvent être strictes (unipolaires du début à la fin de la chaîne opératoire) ou bien au contraire, mixtes en s'adaptant toujours à la morphologie du nucléus dans son processus d'exploitation.

Fig. 8 - Nucléus à éclat préférentiel (négatif en gris) en fin de chaîne opératoire Fig. 8 - Preferential Levallois core

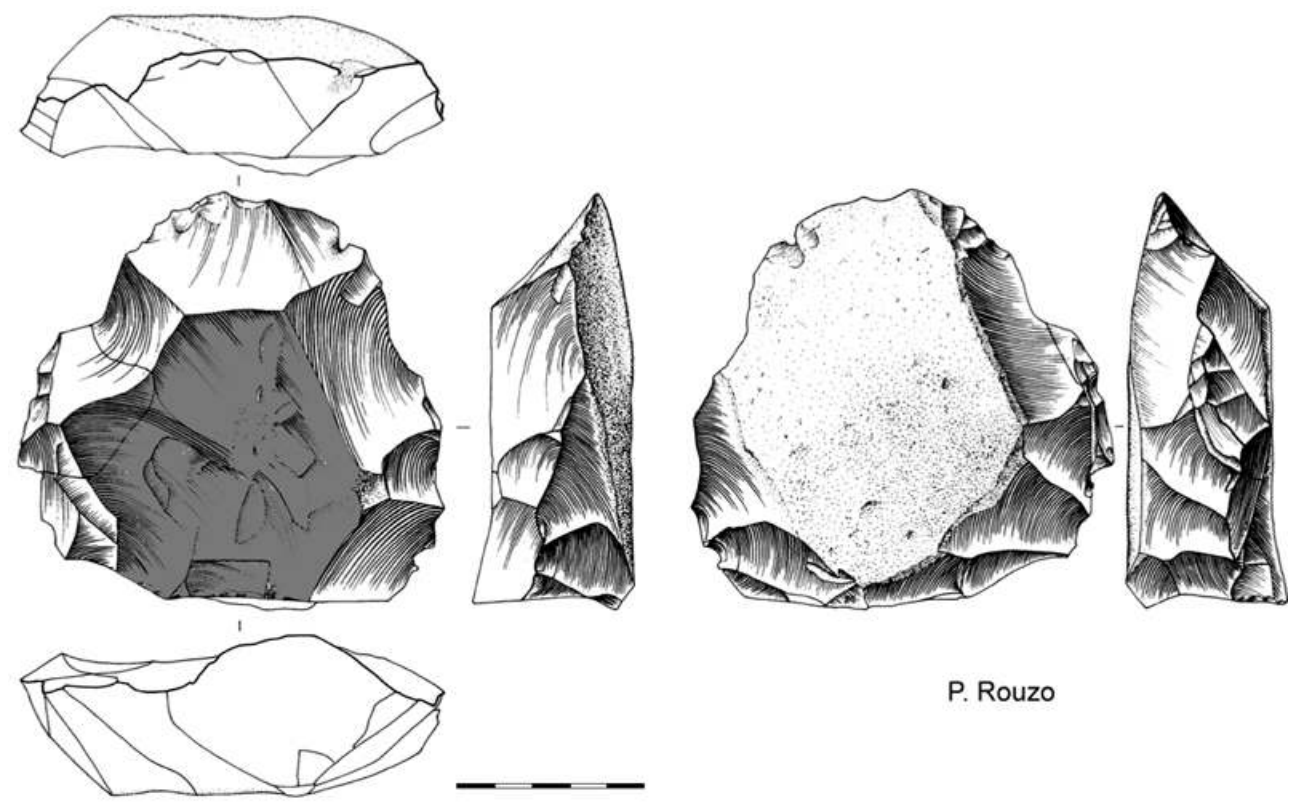


Fig. 9 - Remontage partiel d'un nucléus Levallois de modalité récurrente bipolaire Fig. 9 - Partial refit of bipolar recurrent Levallois core

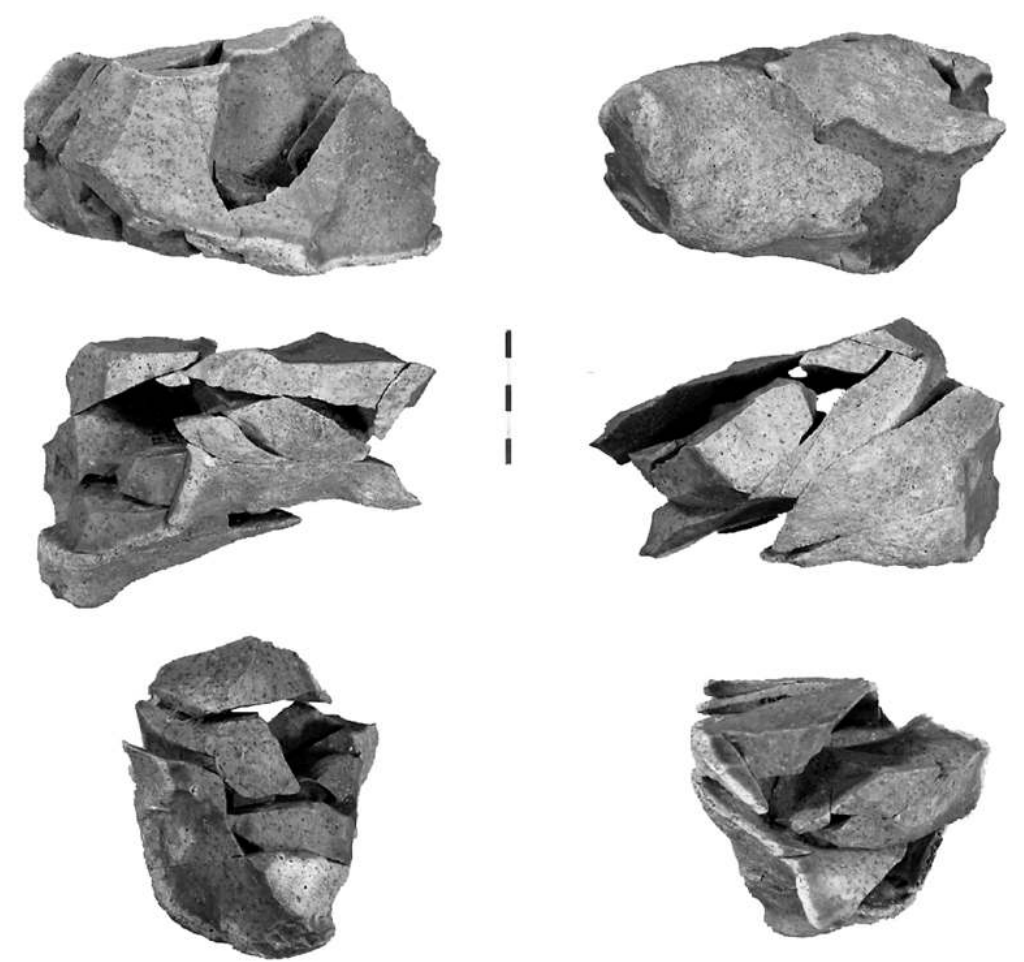

Des disproportions techno-économiques dans la chaîne opératoire de débitage (Geneste 1985) apparaissent :

- en tout premier lieu, il ressort une faible représentation des produits retouchés. Le faible taux de transformation des supports en outils, 35 pièces au total, est une des caractéristiques majeures de cette industrie ;

De plus, à l'intérieur des différentes séquences de production, on remarque également des disproportions. Celles-ci apparaissent d'autant plus fortes lors d'une comparaison avec les données expérimentales obtenues par J.M. Geneste et A. Turq (Geneste op. cit. et Turq 1990) (tabl. : 1, les pourcentages obtenus pour le site de La Folie prenant en compte l'ensemble des produits débités, y compris ceux repris en outils);

- un pourcentage normal voire sur-représenté de produits corticaux selon l'expérimentateur concerné ;

- une sur-représentation des produits à dos corticaux : plus de cinq fois plus que dans les séries expérimentales ;

- une sous-représentation des produits ordinaires ;

- une sur-représentation des produits de préparation de convexité latérale (tels que les débordants) ;

- une sous-représentation des produits Levallois.

Tabl. 1 - Comparaisons entre les pourcentages des différentes catégories technologiques de La Folie et des expérimentations Table 1 - Comparison between the technological categorys and experimentals pourcentages

\begin{tabular}{|l|l|l|l|}
\hline & La Folie & J.-M. Geneste & A. Turq \\
\hline
\end{tabular}




\begin{tabular}{|l|l|l|l|}
\hline éclats corticaux & 33,2 & 29,3 & 12,5 \\
\hline éclats à dos corticaux & 15,5 & 1,8 & 3 \\
\hline éclats ordinaires & 10,5 & 42,5 & 62 \\
\hline éclats Levallois & 11,6 & 17,1 & 17,5 \\
\hline éclats de préparation & 16,1 & 3,7 & 4 \\
\hline Nucléus & 2,82 & 1,1 & 1 \\
\hline Nucléus sur éclat & 1,55 & 0,1 & - \\
\hline éclats «Kombewas» & 4,52 & 0,2 & - \\
\hline éclats de retouche & 0,99 & - & 1 \\
\hline
\end{tabular}

Ce dernier déficit est en général interprété comme une exportation des produits recherchés. Une partie de la production de "La Folie" semble donc avoir fait l'objet d'un transport vers d'autres lieux (ces derniers pouvant être seulement à quelques dizaines de mètres dans un secteur non fouillé ou au contraire être éloignés de quelques dizaines de kilomètres). Certains remontages, parmi les plus complets, où ces supports font défaut, tendent à confirmer cette exportation de produits prédéterminés. Par ailleurs, d'autres remontages confirment l'introduction de blocs de silex préalablement décortiqués, très probablement sur le lieu d'acquisition.

Parmi les supports produits, certains ont été préférentiellement sélectionnés en vue d'être transformés par la retouche. En règle générale, ce sont les supports ayant les plus grandes dimensions, des éclats corticaux ou bien des éclats prédéterminés Levallois qui sont sélectionnés pour être aménagés. Le spectre typologique, dominé par les couteaux à dos retouchés (Fig. 10) et les denticulés, est quelque peu atypique. En l'absence de biface, on ne peut réellement attribuer l'industrie à un Moustérien de Tradition Acheuléenne, bien que ce soit le seul faciès culturel qui puisse être suggéré. 
Fig. 10 - Couteaux à dos abattu sur éclat Fig. 10 - Backed knives on a flake

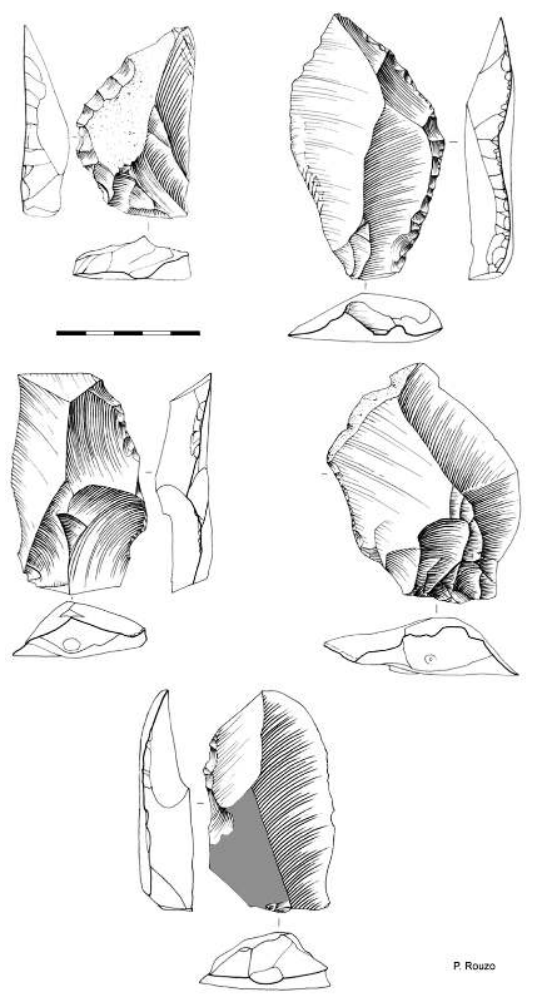

31 S'il est aisé de déterminer et de comptabiliser les outils retouchés, il est impossible dans l'état actuel des connaissances de déterminer le taux de supports bruts potentiellement utilisables sans avoir recours à l'analyse fonctionnelle. Or le test tracéologique mené par S. Beyries sur un échantillon de 46 pièces montre au-delà d'un fort pourcentage d'utilisation ( $30 \%$ de l'échantillon), une utilisation prononcée des éclats bruts (64,2\%) (Bourguignon et al. 2001). Ce n'est donc pas parce que le pourcentage d'outils retouchés est faible, que l'on n'est pas en présence d'une occupation où des activités de transformation et de consommation de biens ont été menées. À "La Folie", celles-ci semblent être assez diversifiées : travail du bois, de la peau et des végétaux souples (Fig. 11). Cependant, on notera, l'absence d'activité liée au travail des carcasses animales (découpe de viande, désarticulation, travail de l'os). Il serait donc intéressant de confirmer cette absence d'activité en réalisant un complément d'étude. 
Fig. 11 - Traces d'utilisation sur les tranchants de supports bruts ou retouchés de La Folie Fig. 11 - Levallois flakes with indication of use-wear traces

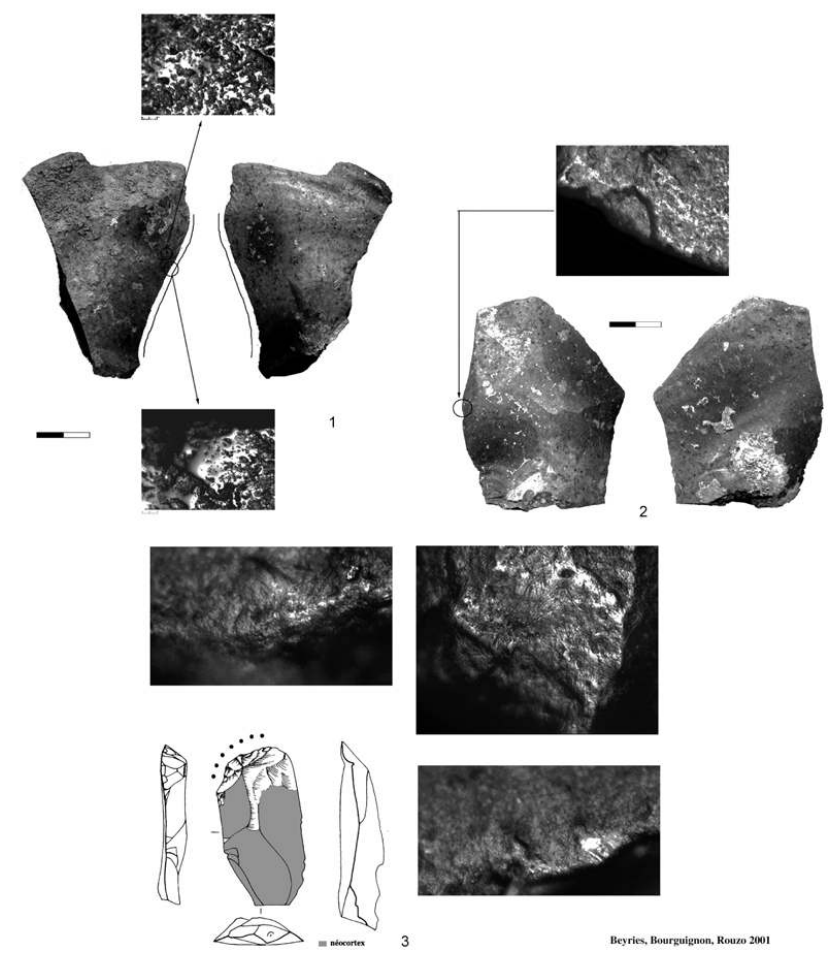

(1) coupe de matière végétale ; (2) découpe de peau sèche (3) grattage de peau sèche vegetal cutting (1), hide cutting (2) and dry hide scraping (3)

Les caractères de l'industrie ne correspondent pas totalement à un faciès de production, ni réellement à un faciès de consommation (trop peu d'outils et de produits Levallois). Ils traduiraient plutôt un faciès mixte de production (allant jusqu'à une exportation de certains types de produits) et de consommation (tranchants bruts et retouchés ayant été utilisés). Nous aurions donc affaire à une production à la fois réalisée pour un usage différé (dans le temps et dans l'espace) et pour un usage immédiat (pendant la durée d'occupation dans le secteur fouillé) comme en témoigne l'étude fonctionnelle.

Cette projection dans le temps de besoins déterminés en terme de prévision suggère une complémentarité d'activités économiques de différents secteurs de l'occupation ou de différentes implantations, ce qui nous permet ainsi de replacer l'occupation de " $\mathrm{La}$ Folie " dans un territoire d'exploitation latent plus large, renvoyant à une organisation de la mobilité des groupes dans cet espace inféré (ce que suggère également l'apport de l'éclat en silex turonien même si c'est en petite quantité). N'oublions pas que le gisement est localisé à la jonction de biotopes différents, d'espaces ouverts (large vallée) et d'espaces cloisonnés (vallées profondes et plateaux) ce qui, dans le cadre d'une acquisition des denrées alimentaires (animales et végétales), a dû jouer un rôle considérable. 


\section{V- Organisation spatiale}

\section{I - Analyse spatiale des remontages lithiques}

Le caractère particulier du site réside dans le fort pourcentage de remontages qui atteint $40 \%$ (en comptabilisant les éclats inférieurs à $2 \mathrm{~cm}$ ) et $62,5 \%$ sans les prendre en considération. Le total des artefacts concernés par ces remontages est de 443 sur 1107 pièces, 62 remontages différents ont pu être réalisés.

Parmi les 29 nucléus dénombrés sur le site, 27 d'entre eux participent aux remontages ce qui représente $93 \%$ du total des nucléus, ce qui semble indiquer que l'essentiel des blocs a fait l'objet d'un débitage sur place. C'est l'ensemble des produits technologiques qui est concerné.

La majorité des remontages est composée de deux artefacts, mais il faut souligner que pour huit d'entre eux, le nombre d'éclats par remontage est supérieur à 10 (soit 12,9\% des remontages). Parmi ces ensembles, sept de ces remontages sont constitués d'un double remontage $(n b=5)$ ou d'un triple remontage $(n b=2$, débitage aux dépens d'éclats).

Les plans de répartition des objets au sol traduisent une dispersion différentielle des vestiges. On observe tout d'abord un aménagement concentrique de blocs calcaires sur une superficie d'environ 10 mètres de diamètre. Ensuite, on note que l'essentiel des vestiges lithiques, subdivisé en deux zones principales, s'inscrit dans cette organisation circulaire. En dehors de cette " structure " (cf. infra), les densités d'artefacts sont plus faibles, voire totalement nulles (Fig. 12).

Fig. 12 - Répartition des vestiges au sol Fig. 12 - Distribution map of all artefacts recorded

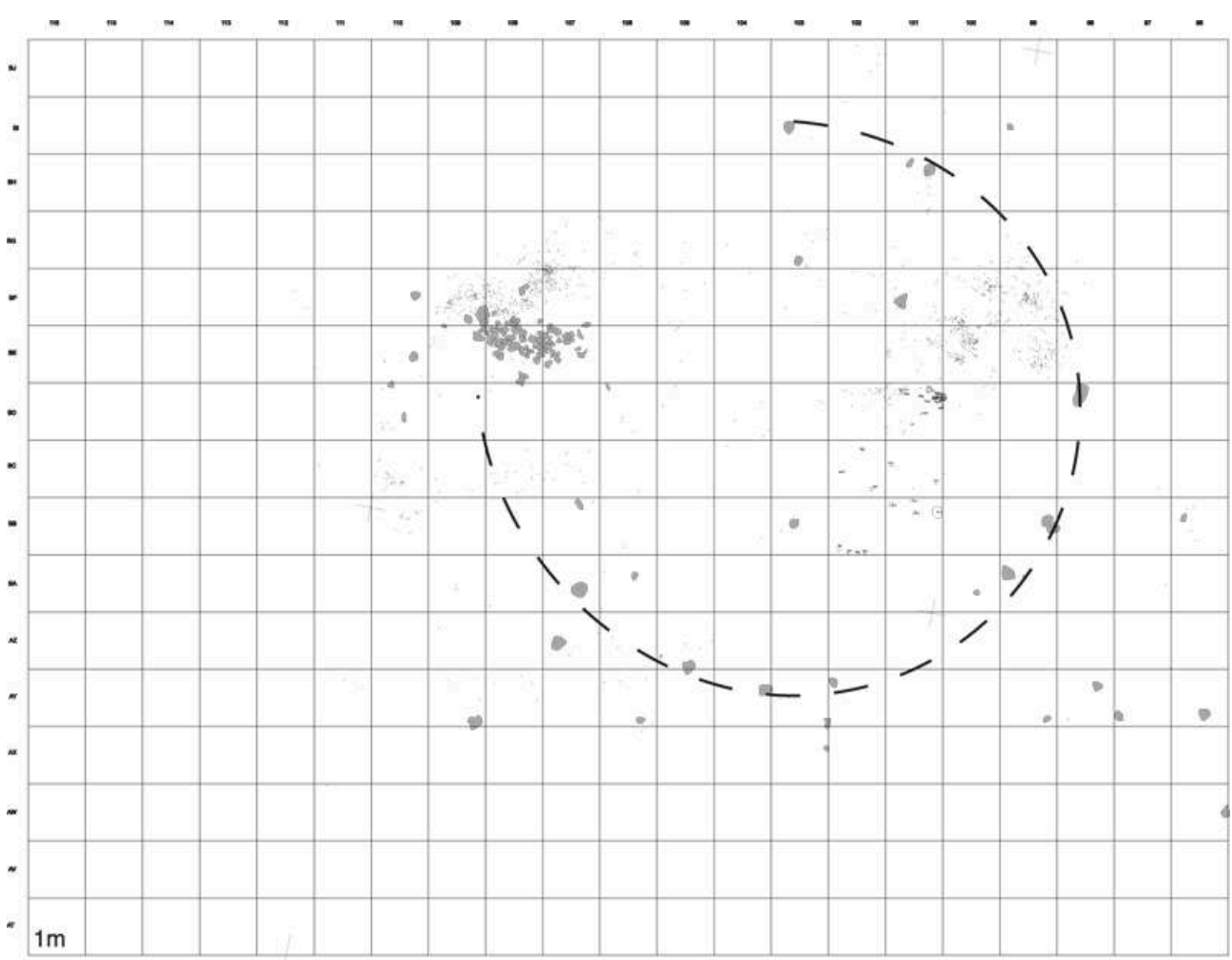


Les plans de densité de l'assemblage lithique montrent clairement l'accumulation de vestiges sous forme d'amas dans les zones est et ouest de la structure et marque ainsi l'emplacement de postes de débitage plus ou moins étendus.

En grande majorité, ces remontages sont “ de proximité " et la répartition des artefacts constituant chacun des groupes de remontage indique à cet emplacement la présence de poste de taille. Cela est d'autant plus probable lorsque le nucléus est présent. Il faut toutefois noter que certains remontages présentent une organisation particulière : les éclats débordants et les éclats à dos corticaux se localisent le plus souvent en périphérie des postes de débitage sans qu'aucune caractéristique technique particulière ne soit observée (Fig. 13).

Fig. 13 - Remontages de proximité (dans les amas) et sur grandes distances (espaces interne et externe) depuis la zone de concentration nord

Fig. 13 - Refits of " proximity" (inside the artefacts concentration) and long distance refits (inside and outside occupation area) since the north concentration area

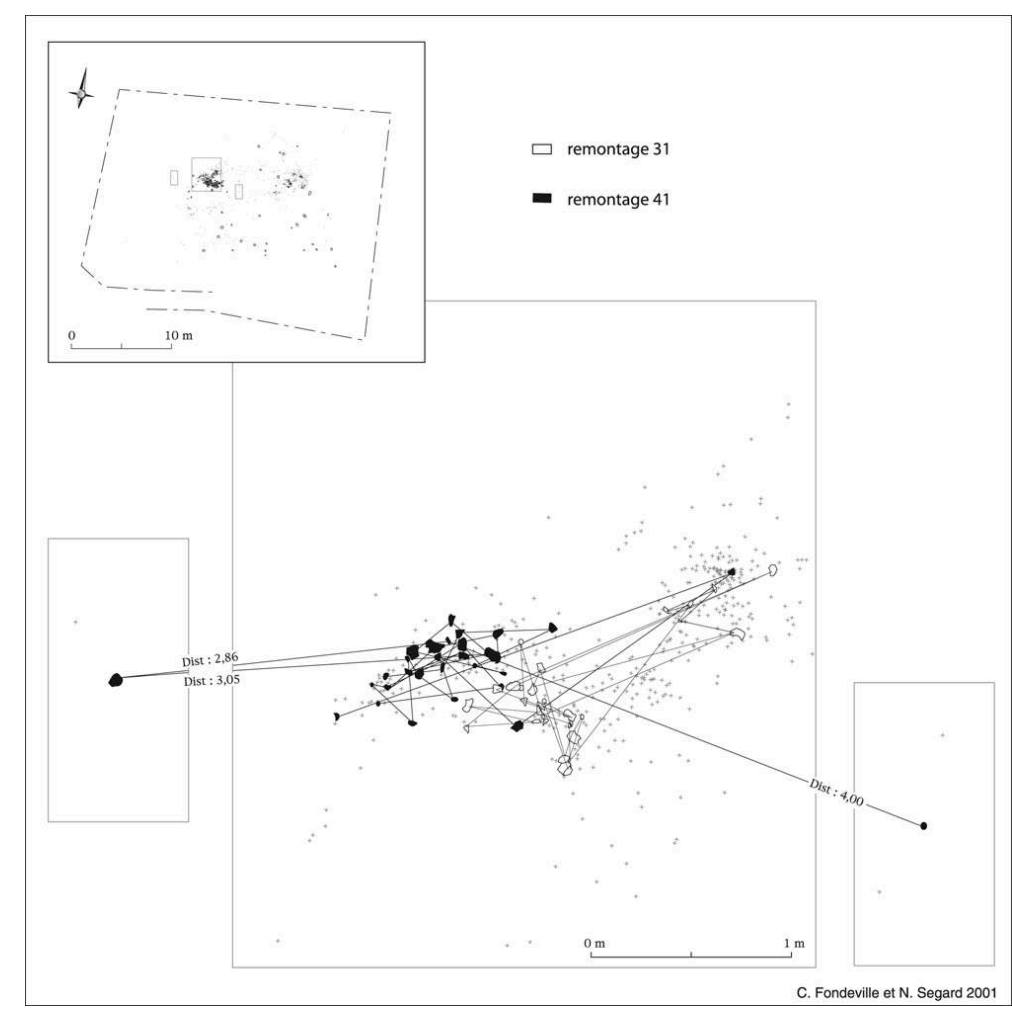

Les remontages sur de grandes distances vers les zones de concentrations est et ouest témoignent du déplacement d'un faible nombre de produits. Ce sont avant tout des nucléus parfois, en cours d'exploitation (continuation du débitage dans un autre lieu), des outils et quelques produits de plein débitage.

41 Les deux secteurs de taille s'organisent autour de deux blocs calcaires. Le premier se localise dans le carré BF. 108 , le bloc pèse $1,8 \mathrm{~kg}$ et présente une forme en "L". Le second, de forme triangulaire, se situe dans le carré BF.101 et pèse $6,9 \mathrm{~kg}$ (Fig. 11). Bien qu'il soit difficile d'affirmer qu'il s'agisse véritablement d'un pôle intentionnel du "siège du tailleur", la forme particulière des blocs et celle de leur isolement à proximité des amas de débitage pourrait néanmoins suggérer cette hypothèse ${ }^{2}$.

42 V.II- Analyse spatiale des blocs calcaires 
Dans la zone ouest du gisement, non loin de la plus forte concentration de silex, 85 pierres se jouxtent pour former un empierrement allongé de deux $\mathrm{m}$ de longueur environ sur $65 \mathrm{~cm}$ de large (Fig. 14a). Latéralement, quatre pierres se détachent de la concentration: trois sont regroupées au sud, et une seule se localise au nord, en bordure de la zone de rubéfaction mise en évidence par la micromorphologie. Le poids des blocs formant cet empierrement est compris entre 0,1 et 1,6 kg.

Fig. 14 - Localisation de la zone empierrée, des amas et de la zone rubéfiée Fig. 14 - Location of stones concentration, artefacts concentration and burned area

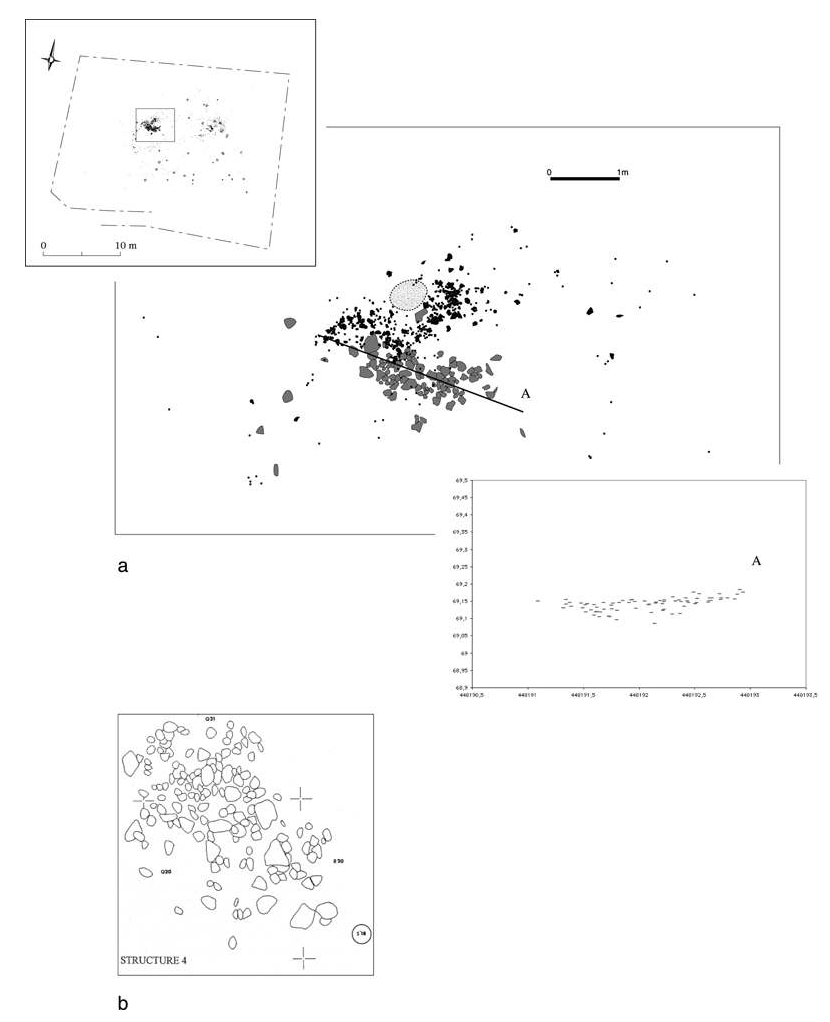

(a) et structure 4 (b) du gisement de Marolles-sur-Seine (d'après Julien et Rieu 1999)

(a), structure 4 (b) of Marolles-sur-Seine site (Julien et Rieu 1999)

Cet empierrement semble être dû à une accumulation volontaire en nappe peu dispersée, accumulation pouvant elle-même résulter du déplacement des pierres après leur usage ou pour leur usage.

47 Les différentes coupes réalisées, notamment la longitudinale (Fig. 14a), suggère la présence d'une légère dépression au sein de laquelle viennent se disposer les pierres de façon presque jointives. Ce sont essentiellement des pierres de petits modules et régulièrement de section plano-convexe qui ont été sélectionnées. Un encroûtement 
calcitique vient souvent recouvrir ces éléments calcaires, voire même les sceller les uns aux autres. abondance d'objets lithiques sous la forme d'un gros amas bilobé (voire de deux sousamas de débitage). Ces vestiges lithiques ont une répartition asymétrique : toujours d'un côté ou de l'autre des deux structures (Fig. 14a). Cette distribution des vestiges autour des foyers est par ailleurs assez fréquente, que ce soit pour des gisements du Paléolithique inférieur (Port Pignot et la Roche Gelatan ${ }^{3}$, du Paléolithique moyen (Saint-Germain-des Vaux ${ }^{4}$ ou encore Paléolithique supérieur (Pincevent, Marsangy, Etiolles). Lieu de consommation, de source de chaleur et de lumière, le foyer constitue en effet un des espaces privilégiés de l'unité domestique préhistorique.

\section{Agencement $1:$ une habitation circulaire moustérienne ?}

C'est en s'appuyant sur la définition proposée dans le dictionnaire de la Préhistoire que nous tenterons d'étayer l'hypothèse d'une habitation sur le gisement de "La Folie" : “ structure aménagée par les hommes pour constituer la partie centrale d'un site d'habitat, et dont les vestiges permettent de mettre en évidence qu'elle réalisait une opposition entre un espace intérieur, généralement abrité, et l'espace extérieur. L'intérieur d'une habitation est utilisé pour une partie des activités domestiques, et dans tous les cas, par définition pour le sommeil." (Leclerc et Tarrête 1994 p. 482-483).

54 Les données de terrain et les premières interprétations 
Si nous relions le maximum de blocs calcaires compris entre 1 et $19 \mathrm{~kg}$ (13/23) (Fig. 15, blocs $n^{\circ} 1$ à 13), il semble se dessiner une forme circulaire de grand diamètre. Trois blocs supplémentaires (blocs $\mathrm{n}^{\circ} 14,15,17$ ) pourraient également faire partie de cette matérialisation si l'on suppose un léger déplacement vers l'extérieur. Ce cercle presque parfait pourrait constituer la matérialisation des limites externes d'une habitation.

Fig. 15 - Répartition des blocs (en noir) sur la surface d'occupation moustérienne Fig. 15 - Map distribution of stones (in black) in excaved area of the mousterian site of La Folie

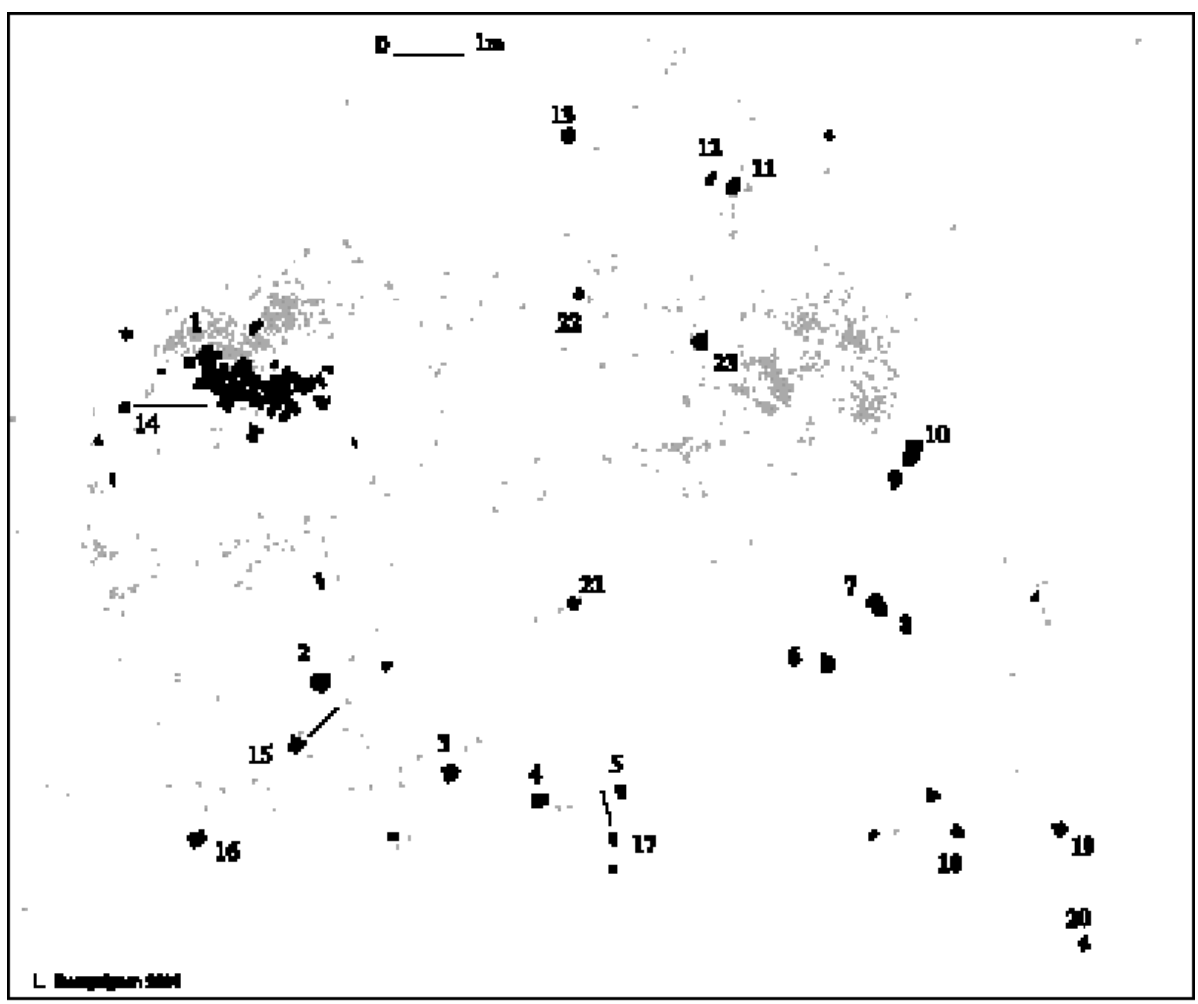

Plusieurs observations le suggèrent :

- les analyses micro-morphologiques montrent clairement que ces blocs ne peuvent avoir ni une origine ni une disposition naturelle (Sellami 2001). Cela induit que ces blocs calcaires ont été apportés intentionnellement dans la station, qu'ils ont pu faire l'objet d'une sélection (pour leur poids ?) et que leurs emplacements et disposition ne sont pas aléatoires ;

- la forme même de cet agencement : un cercle parfait de grand diamètre ;

- la présence d'un trou de poteau avec calage (Fig. 15 blocs $n^{\circ} 7$ et 8) suggère un système d'ancrage d'une armature et/ou d'une couverture d'une habitation. Il se localise parfaitement dans la matérialisation du cercle, dans sa partie ouest ;

- une organisation topographique interne à cette délimitation circulaire. Les zones de concentration de vestiges de fortes densités (amas de taille) sont localisées, soit à l'intérieur de ce périmètre (concentration II à V ; Fig. 16), soit en limite externe/interne (concentration I ; Fig. 16). Quelques objets épars ou constituant des zones de concentration de faible densité se situent essentiellement dans un périmètre rapproché des concentrations principales ( $\mathrm{b}$ II b et IV b ; Fig. 16). 
Fig. 16 - Plan synthétique des différentes zones de densité du mobilier Fig. 16 - Synthetic map of the differents area artefacts concentrations

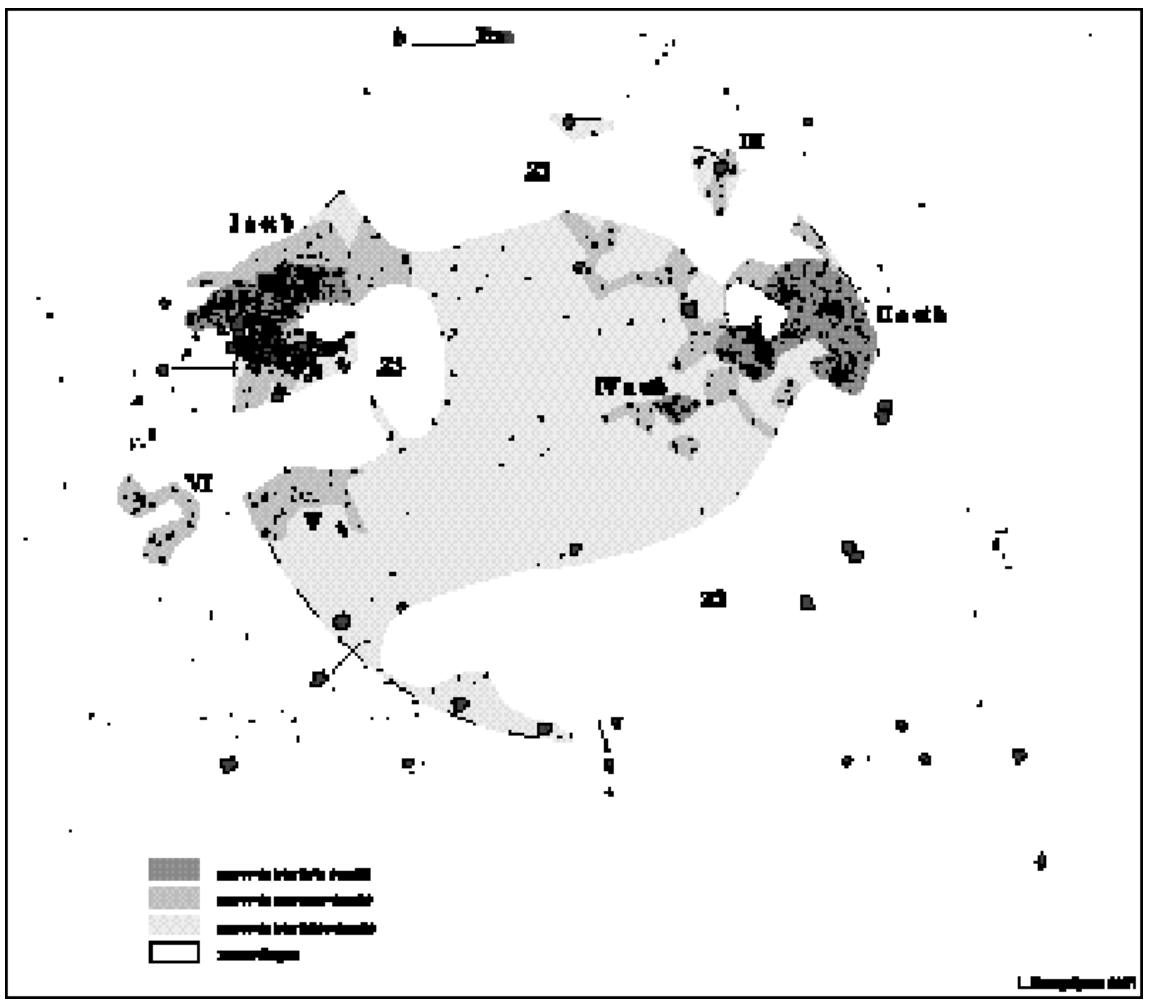

57 L'essentiel des remontages sur longues distances relie les deux grandes zones de concentrations internes selon un axe approximatif est-ouest (Fig. 17). Néanmoins, les axes de remontages sur de longues distances montrent un comportement légèrement différent entre ces deux zones. La majorité des remontages sur de longues distances de la concentration I est orientée vers l'intérieur de la structure (notamment vers la concentration II) alors que ceux de la concentration II mettent plus souvent en relation des vestiges isolés localisés à l'extérieur (vers le nord). À l'inverse, les liaisons internes de ces différentes concentrations sont courtes. Paramètres qui d'après A. Leroi Gourhan "circonscrivent en quelques mètres carrés les limites de l'espace domestique, joignant le foyer aux amas de déchets les plus proches" (Leroi Gourhan 1983, p. 170) ;

- cette matérialisation circulaire s'ajuste souvent avec une limitation plus ou moins franche des concentrations de silex. C'est le cas pour les concentrations nord-est II et III et la concentration ouest V (Fig. 16). Ces délimitations pourraient laisser suggérer la présence d'un obstacle empêchant une dispersion latérale plus vaste des objets. Ces possibles effets de parois sont néanmoins à considérer avec précaution car des amas de taille en place gardent également des limites nettes. Les expérimentations menées par E. Boëda et J. Pelegrin pour reconstituer les amas de taille de Marsangy et tester l'hypothèse d'effet de parois sur ce gisement ont montré que des limites nettes observables n'impliquent pas forcément la présence d'un obstacle (Boëda et Pelegrin 1985) ;

- dans cet espace interne, des zones vierges de tout vestige se distinguent très nettement. La présence de matières périssables, comme des peaux, du bois et des végétaux, voire des ossements dans certains cas, laissent de telles signatures. À “La Folie ”, les espaces vides les plus significatifs se localisent, soit à proximité immédiates des zones de concentrations (Fig. 16 zones vierges $\mathrm{Z} 1$ et Z3), soit dans la zone qui pourrait être considérée comme le fond de l'habitation (Fig. 16 zone vierge Z2). Les premières pourraient être interprétées comme 
des zones de traitement ou de consommation des matières périssables, la seconde comme une zone de repos recouverte d'un tapis organique. Ce dernier, comme plusieurs échantillons micro-morphologiques l'ont démontré, est végétal (cf. supra et Sellami 2001). L'emplacement de cette couverture végétale correspond bien aux modèles ethnographiques où le fond de la tente est toujours occupé par la literie. Sur le gisement de Pincevent, l'emplacement des "couches de repos" est également localisé au fond de la tente, elles se distinguent du reste de l'habitation par une aire non teintée par l'ocre (Leroi Gourhan op. cit. p. 190-191).

Fig. 17 - Plan de répartition des remontages totaux Fig. 17 - Map of rifits

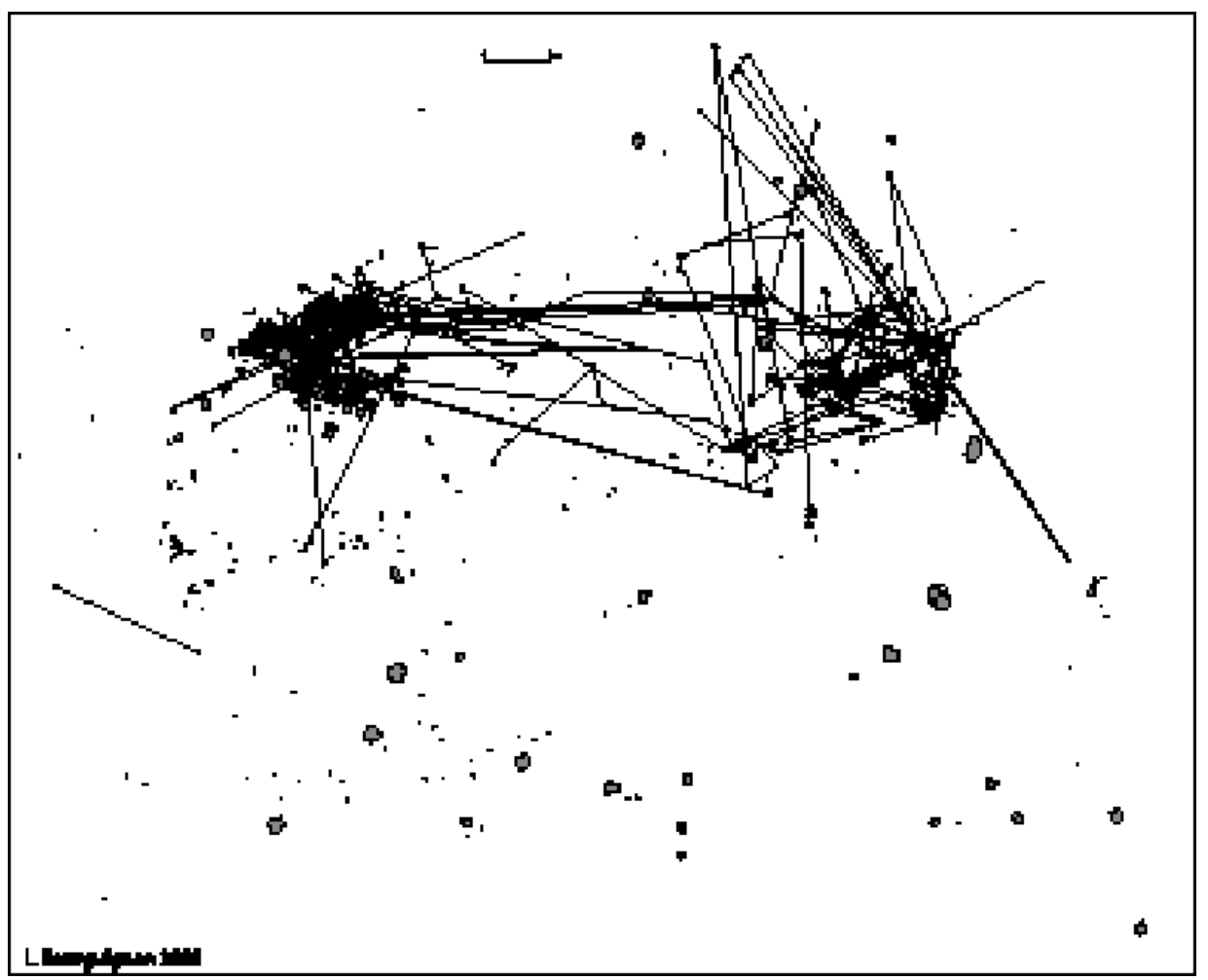

C'est à l'opposé de cette zone de repos, selon un axe sud-est/nord-ouest, vers l'ouverture supposée, que se localiserait le secteur de combustion. Localisation également compatible avec les modèles ethnographiques connus, où les foyers peuvent être placés à l'intérieur (indiens d'Amérique du Nord), devant la construction (Onea de la Terre de feu) devant l'entrée (Boschimans d'Afrique du Sud) ou encore sur un côté près de l'entrée (Esquimos Caribou).

- l'espace externe se différencie de l'espace interne par une rareté de vestiges. Ils sont le plus souvent isolés, hormis une petite concentration à l'ouest (Fig. $16 \mathrm{n}^{\circ} \mathrm{VI}$ ). Cette dernière est d'ailleurs assez particulière puisqu'elle est essentiellement constituée d'éléments inférieurs à trois $\mathrm{cm}$ et qu'elle pourrait correspondre à un poste spécialisé dans l'aménagement d'outils (Segard 2001). On notera également la présence de quelques blocs de calcaires (compris entre 0,3 et $3,9 \mathrm{~kg}$ ) et un bloc de silex (de $2 \mathrm{~kg}$ ) isolés de tout autre vestige et localisés en marge sud-est de l'occupation. Cette raréfaction des vestiges abandonnés autour de l'habitation concourt dans de nombreux cas à la définition de sa forme (cas de Pincevent et d'Etiolles par exemple). 
point de vue méthodologique, l'étude abordée ici montre bien la nécessité d'appréhender les gisements de cette période selon des problématiques paléoethnographiques y compris en contexte de sauvetage. Elle souligne également le besoin d'enregistrer sur le terrain de très nombreuses informations selon la complexité des phénomènes et le développement d'analyses susceptibles d'intervenir postérieurement. Malheureusement, ce type d'analyses ne peut être que rarement prévu en amont, ce n'est qu'en cours de fouille (au plus tôt) que l'implication de telles informations semble nécessaire à la compréhension d'agencements pourtant incontestablement évidents.

Le problème majeur rencontré lors de cette approche de la structuration d'un espace " habité clos " réside dans le manque important de références comparatives pour cette période chronologique. Si les premières habitations connues sont attribuables au Paléolithique inférieur (muret de pierres d'olduvaï, habitation de Terra Amata), durant le Paléolithique moyen les traces de structures d'habitation tangibles nous manquent (hormis Molodova en Ukraine). Pourtant, certains agencements particuliers de blocs auraient pu donner suite à des développements interprétatifs plus avancés. C'est le cas, par exemple, de la structure circulaire du gisement moustérien de la Butte d'Arvigny (Gouédo et al. 1994). C'est donc essentiellement vers le corpus de données du Paléolithique supérieur, beaucoup plus riche, que nous avons orienté nos recherches documentaires. Celles-ci demanderaient à être plus largement élargies et approfondies, de même que pour le référentiel ethnographique actualiste. Les mêmes types de remarques nous empêchent de tirer de quelconques conclusions sur les autres structures potentielles du gisement.

61 Aussi, les résultats et interprétations contenus dans cet article sont à considérer comme des hypothèses constituant des axes privilégiés de recherche que nous nous sommes fixés. Nous espérons pouvoir les développer et les argumenter plus profondément lors d'un travail ultérieur, dans le cadre d'une monographie.

\section{VI- Conclusions}

62 Le gisement moustérien de "La Folie " à Poitiers constitue un référentiel important dans le contexte régional de cette période. La très grande rareté des données concernant les occupations du Paléolithique moyen dans la Vienne fait du niveau d'habitat moustérien de "La Folie " un gisement d'un grand intérêt. En effet, les rares découvertes régionales du Paléolithique moyen de plein air se réduisent le plus souvent à des ramassages de surface. Quelques gisements en grotte ou sous abri sont toutefois présents dans les vallées principales et secondaires du département (vallées du Clain, de la Vienne, de l'Anglin et de la Creuse). Le faciès culturel le plus représenté régionalement est le Moustérien de Tradition Acheuléenne, ce qui, nous l'avons vu, malgré un caractère atypique, correspondrait le mieux à l'attribution de l'industrie de “ La Folie".

63 D'un point de vue technologique et typologique, l'analyse du matériel lithique a montré des caractéristiques qui semblent attribuables à ce faciès. De débitage Levallois, l'industrie se caractérise par un outillage dominé par les couteaux à dos (retouchés et naturels) et les denticulés avec une absence de biface.

PALEO, 14 | 2002 
64 du groupe implanté sur les berges du Clain. Ils illustrent une exploitation du milieu minéral local (exception faite du milieu alluvial) par une acquisition de blocs de silex et de calcaire sur les versants avoisinant le campement. Les blocs de silex sont exploités selon une conception Levallois de modalités récurrentes pour l'essentiel. Ces modalités sont adaptées à la configuration des blocs. Bien que les supports produits soient peu aménagés par la retouche, l'utilisation de la production est attestée à la fois sur des outils retouchés et sur des supports bruts. Des activités de découpe et de grattage sur diverses matières d'œuvre (peau, bois, végétal souple) ont été menées durant l'occupation, ce qui laisse suggérer qu'une part importante de la production a été réalisée pour des besoins immédiats. L'exportation de certains produits est également avérée, laissant supposer des besoins différés (dans le temps et dans l'espace) ou d'autres aires d'activités contemporaines avoisinantes.

D'un point de vue paléoethnographique, ce gisement constitue un référent rare et idéal, bien que pour l'heure l'ensemble des données recueillies ne soit pas intégralement interprétable. Le niveau d'occupation, enfoui sous une accumulation sédimentaire d'une puissance de deux m, est compris dans des limons fins de débordement. Leur mise en place par un cours d'eau méandriforme à faible compétence a permis une préservation exceptionnelle des vestiges pour cette période grâce à un recouvrement rapide. Ainsi, la structure de cellules végétales, pourtant très fragile, a pu être préservée, ce qui sous-entend une absence de dynamique sédimentaire postdépositionnelle et une préservation parfaite du niveau. De ce fait, l'ensemble des vestiges compris dans ce niveau n'a que très peu subi de remaniements (légers transferts latéraux et activité biologique) et leur disposition est quasi originelle. Cette distribution au sol des vestiges traduirait une organisation de l'espace à l'intérieur d'une structure circulaire qui pourrait correspondre aux restes d'une habitation. Ces limites se matérialisent par un agencement de gros blocs sous forme de cercle, parmi lesquels un trou de poteau avec calage a pu être déterminé. À l'intérieur de cette structure circulaire, des zones de concentrations, de déchets de production pour l'essentiel (amas de débitage), s'opposent à des zones vierges dont plusieurs peuvent être interprétées comme des empreintes de matériaux périssables (végétaux). Certaines signatures démontrent en effet l'existence d'un tapis végétal associé à la localisation d'une grande zone vierge dans le fond de l'habitation qui peut suggérer l'existence d'une aire de repos.

D'un point de vue chronologique, aucune datation absolue n'a pu être entreprise. Si l'on émet l'hypothèse que la nappe alluviale sous-jacente au niveau archéologique peut se rattacher à la glaciation weichsélienne dans un contexte climatique rigoureux (stades isotopiques $5 \mathrm{~d}$ à $5 \mathrm{~b}$ ), alors les limons fins de débordement, qui scellent l'occupation moustérienne, pourraient être attribués à une phase climatique plus tempérée de cette glaciation (stade isotopique $5 \mathrm{a}$ ). Cette hypothèse chronologique basée sur les données géologiques placerait l'occupation entre 84000 et 72000 B.P. Bien que les analyses palynologiques ne permettent pas de reconstituer le contexte paléo-environnemental, certains faits s'accorderaient avec cet épisode climatique froid et l'occupation aurait pu avoir lieu lors de la régression de la pinède. Les hommes auraient ainsi pu bénéficier à la fois de l'abri de la forêt et de l'extension d'espaces découverts plus favorables au passage du gibier. Les échantillons malacologiques prélevés, en cours d'étude, pourront peut-être compléter cette reconstitution climatique.

PALEO, 14 | 2002 
La faible densité de matériel lithique, débitage d'un maximum de 13 blocs répartis sur une épaisseur maximale de $10 \mathrm{~cm}$ sur toute la surface, associé à un très fort taux de remontage laisse suggérer une unique occupation. Celle-ci, selon les activités menées (débitage et utilisation sur place des objets) et les investissements engagés (manuport des blocs calcaires pour la constitution des structures agencées et leur réalisation), devait occuper une place privilégiée dans l'économie des chasseurs-cueilleurs. L'absence de faune nous prive malheureusement d'un pan entier des activités alimentaires et techniques. Elle aurait pu nous aider plus justement à l'identification de la spécificité du site. De même, on ne peut pas réellement évaluer la taille du groupe puisque le secteur fouillé, bien qu'étant homogène et formant une unité, semble faire partie d'un complexe beaucoup plus grand.

L'un des points positifs de cette étude est très certainement d'avoir montré qu'il pouvait exister durant le Moustérien des contextes très favorables à l'enregistrement de diverses activités anthropiques et que ces activités traduisaient vraisemblablement une organisation économique et sociale plus complexe qu'on ne l'admettait jusqu'alors. À ce titre, il est fort regrettable de n'avoir pu développer les recherches de terrain sur ce gisement. L'archéologie préventive, bien qu'elle permette la découverte de ce type de site exceptionnel et l'enregistrement de nombreuses données en un temps réduit, constitue souvent un obstacle (faute de temps et/ou de moyens) à la connaissance des comportements socio-économiques des groupes humains. En effet, l'extension des surfaces fouillées est limitée en surface à l'emprise du projet, cette contrainte peut dans certains cas restreindre la compréhension générale du gisement ou interdire des interprétations.

\section{BIBLIOGRAPHIE}

BOËDA E. et PELEGRIN J. 1985 - Approche expérimentale des amas de Marsangy. In : Les amas lithiques de la zone N19 du gisement Magdalénien de Marsangy : approche méthodologique par l'expérimentation. Archéologie Expérimentale, cahier n 1, Archéodrome 1985, pp. 19-36.

BOURGEUIL B., CARIOU E., MOREAU P., DUCLOUX J., 1976 - Notice explicative de la feuille de Vouneuil-sur-Vienne (567) à 1/50 000. Ed. BRGM, Orléans, 24 p.

BOURGUIGNON L. et ORTEGA I. 1998 - Les Champs de Bossuet. In : Bilan scientifiquedu Service Régional de l'Archéologie d'Aquitaine 1998, p. 146-147.

BOURGUIGNON L. et ORTEGA I. 2000 - Le gisement moustérien de Champs de Bossuet, DFS, Service Régional de l'Archéologie d'Aquitaine.

BOURGUIGNON L., SEGARD N., DELOZE V., SELLAMI F., EMERY-BARBIER A. et BEYRIES S. 2001 - Le gisement moustérien de "La Folie " Poitiers, DFS, Service Régional de l'Archéologie de PoitouCharentes.

CAMPY M. et MACAIRE J.-J. 1989 - Géologie des formations superficielles : géodynamique, faciès, utilisation. Ed. Masson, Paris, 1989, 433 p. 
CLIQUET D., DUMONT J.-L., DUPONT J.-P., FOSSE G., JOUIS M., LANOE B., MANTE C., MOREL J., QUILLARD J., THIEBAULT S. et VILGRAIN G. 1989 - Approche d'une étude comparative des matières organiques des foyers préhistoriques et des foyers expérimentaux. L'exemple du gisement moustérien de Saint-Germain-des-Vaux. In : Actes du colloque de Nemours 1987, Mémoires du Musée de Préhistoire d'Ile de France, 2. pp. 29-36.

COUDRET P., LARRIERE M. et VALENTIN B. 1989 - Comparer les foyers : une entreprise difficile. In : Actes du colloque de Nemours 1987, Mémoires du Musée de Préhistoire d'Ile de France, 2. 1989. pp. 3745.

DELOZE V. 2001 - Etude géologique et géomorphologique. In : Le gisement moustérien de "La Folie" Poitiers, L. Bourguignon, N, Segard, V Deloze, F Sellami , A. Emery-Barbier et S. Beyries, DFS, Service Régional de l'Archéologie de Poitou Charentes, p. 7 à 36.

FREYTET P., 1992 - Les cristallisations de calcite associées à des restes végétaux (algues, feuilles) en milieu fluviatiles et lacustre, actuel et ancien (tufs et travertins). In : Bull. Soc. Bot. Fr., 139, actuel. Bot. (1), 69-74.

GENESTE. J.-M. 1985 - Analyse lithique d'industries moustériennes du Périgord: Une approche technologique du comportement des groupes humains au Paléolithique moyen. Thèse de l'Université de Bordeaux I, 2 vol. , 567 p.

JULIEN M. et RIEU J-L. 1999 - Occupations du Paléolithique supérieur dans le Sud-Est du Bassin Parisien. Documents d'Archéologie française 78, Série archéologie préventive - Autoroutes A5/A160 ; $236 \mathrm{p}$.

LEBRET P., CAMPY M., COUTARD J.-P., FOURNIGUET J., ISAMBERT M., LAUTRIDOU J.-P., LAVILLE P., MACAIRE J.-J., MENILLET F., MEYER R. 1993 - Cartographie des formations superficielles. Réactualisation des principes de représentation à 1/50 000. Géologie de la France, n 4, 1993 , p. 39-54, 4 fig.

LECLERC J.-L. et TARRETE J. 1994 - Définitions de "habitat" et "habitation". In : Dictionnaire de la Préhistoire. A. Leroi Gourhan, Presses Universitaire de France, 2e édition, 1994, Mai, Paris, p. 482-4823.

LENOBLE A., ORTEGA I. et BOURGUIGNON L. 2000 - Processus de formation du site Moustérien de Champs de Bossuet (Gironde), In : PALEO n 12, p. 413-425.

LEROI-GOURHAN A. 1983 - Le fil du Temps. Ethnologie et préhistoire. Librairie A. Fayard 1983. 318 p.

MICHEL D. 1989 - Les foyers du Paléolithique inférieur du Nord Cotentin. In : Actes du colloque de Nemours 1987, Mémoires du Musée de Préhistoire d'Ile de France, 2. pp. 131- 139.

MOURIER J.-P., GABILLY J., PLATEL J.-P. 1986 - Notice explicative de la feuille de Poitiers (589) à 1/50 000. Ed. BRGM, Orléans, $47 \mathrm{p}$. Notice explicative de la feuille de Vouneuil-sur-Vienne (567) à 1/50 000. Ed. BRGM, Orléans, 1976, 24 p.

SEGARD N. 2001 - Analyse spatiale de l'industrie lithique. In : Le gisement moustérien de "La Folie" Poitiers, L. Bourguignon, N, Segard, V Deloze, F Sellami , A. Emery-Barbier et S. Beyries, DFS, Service Régional de l'Archéologie de Poitou Charentes, pp. 85-116.

SELLAMI F. 2001 - Etude microstratigraphique et de l'organisation du niveau d'occupation du site Moustérien de La Folie. Rapport de l'étude micromorphologique. In : Le gisement moustérien de "La Folie" Poitiers, L. Bourguignon, N, Segard, V Deloze, F Sellami , A. Emery-Barbier et S. Beyries, DFS, Service Régional de l'Archéologie de Poitou Charentes, p. 37 à 52.

TABORIN Y. 1989 - Le Foyer : document et concept. In : Actes du colloque de Nemours 1987, Mémoires du Musée de Préhistoire d'Ile de France, 2. 1989. pp. 77-80. 
TCHEREMISSINOF Y., FOUERE P. et SALANOVA L. 2000 - La sépulture campaniforme de La Folie (Poitiers, Vienne) : présentation préliminaire. Actes des Journées d'Information, Internéo 3, 2000, p. 161-168.

TCHEREMISSINOF Y., BRAGUIER S., CONVERTINI F., DELOZE V., FOUERE P., MIAILHE V., RANCHE C., SALANOVA L. 2001 - “La Folie ” occupations néolithiques et protohistoriques. DFS de sauvetage urgent, AFAN, Service Régional de l'Archéologie de Poitou-Charentes, 2001, 113 p.

TURQ A. 1990 - Exploitation du milieu minéral : Technologie, économie et circulation des matières premières. In : Les chasseurs d'Aurochs de La Bord, un site du Paléolithique moyen (Livernon, Lot) par J. Jaubert, M. Lorblanchet, H. Laville, R Slott-Moller, A Turq et J.-P Brugal. DAF n 27. Edition de la maison des Sciences de l'Homme Paris, 1990. pp. 104-114.

\section{NOTES}

1. d'après le code des couleurs des sols d'A. Cailleux (Editions Boubée).

2. Un autre gisement moustérien de plein air, Champs de Bossuet (Gironde), a livré un siège de tailleur en contexte périphérique d'un amas de taille (Bourguignon et Ortega 1998, 2000).

3. D. Michel 1989

4. D. Cliquet et al. 1989

\section{RÉSUMÉS}

Le gisement moustérien de La Folie illustre un campement de plein air en bordure du Clain d'une rare qualité de préservation. Plusieurs structures ont été mises au jour (structures empierrées, zone de combustion, trou de poteau avec calage...) révélant une organisation de l'espace claire, avec des zones d'activités bien délimitées. Ce gisement constitue un exemple exceptionnel pour cette période en Europe occidentale.

The Mousterian open-air site of La Folie in the Clain valley is a rare exemple of high quality of preservation. Several features and finded (stones structure, hearth,...) which caracterize a clear spatial organization with well delimited activities areas. This site is an exceptional model for these period in occidental europe.

\section{INDEX}

Keywords : Mousterian, Levallois, habitation, hearth, post-hole

Mots-clés : Moustérien, Levallois, habitat, foyer, trou de poteau 
AUTEURS

L. BOURGUIGNON

INRAP, direction inter-régionale Grand Sud-Ouest, 33600 Pessac

F. SELLAMI

INRAP, INA-PG, DMOS, 78850 Grignon

V. DELOZE

INRAP, direction inter-régionale Grand Sud-Ouest, 33600 Pessac

N. SELLIER-SEGARD

NRAP, direction inter-régionale Nord Picardie, 80000 Amiens

S. BEYRIES

CNRS, CEPAM Sophia Antipolis, 06560 Valbonne

A. EMERY-BARBIER

UMR 7041, Maison de l'Archéologie et de l'Ethnologie, 92000 Nanterre 